\title{
Parameterization of the Extinction Coefficient in Ice and Mixed-Phase Arctic Clouds during the ISDAC Field Campaign
}

\author{
A Korolev \\ A Shashkov \\ H Barker
}

March 2012

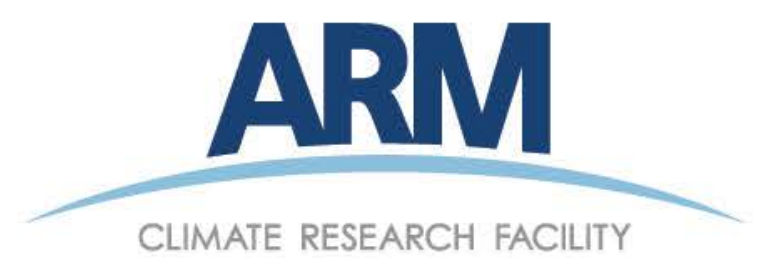




\section{DISCLAIMER}

This report was prepared as an account of work sponsored by the U.S. Government. Neither the United States nor any agency thereof, nor any of their employees, makes any warranty, express or implied, or assumes any legal liability or responsibility for the accuracy, completeness, or usefulness of any information, apparatus, product, or process disclosed, or represents that its use would not infringe privately owned rights. Reference herein to any specific commercial product, process, or service by trade name, trademark, manufacturer, or otherwise, does not necessarily constitute or imply its endorsement, recommendation, or favoring by the U.S. Government or any agency thereof. The views and opinions of authors expressed herein do not necessarily state or reflect those of the U.S. Government or any agency thereof. 


\title{
Parameterization of the Extinction Coefficient in Ice and Mixed-Phase Arctic Clouds during the ISDAC Project
}

\author{
A Korolev \\ A Shashkov \\ H Barker
}

March 2012

Submitted to the:

U.S. Department of Energy

Atmospheric Sciences \& Global Change Division

Pacific Northwest National Laboratory

Richland, WA 99352 USA

Contract Number 143648

Environment Canada

Cloud Physics and Severe Weather Research Section, 4905 Dufferin Street, Toronto,

Ontario, M3H 5T4, Canada 


\section{Contents}

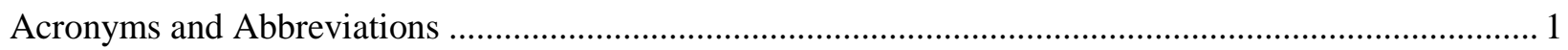

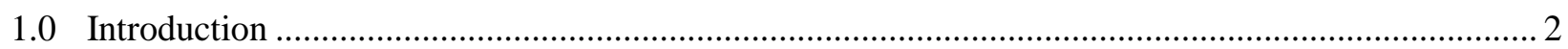

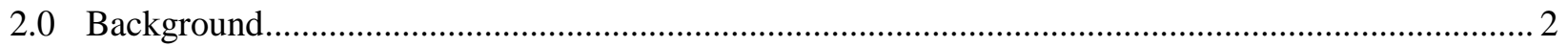

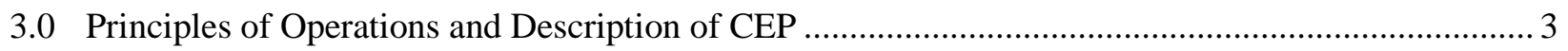

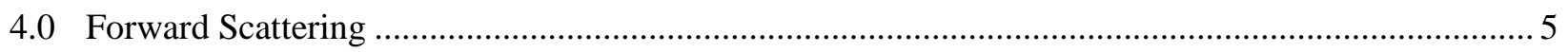

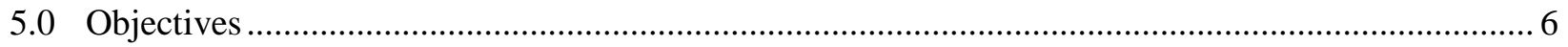

6.0 Effect of Forward Scattering on the Extinction Coefficient Measurements...................................... 6

6.1 Theoretical Calculations of the Effect of Forward Scattering................................................ 6

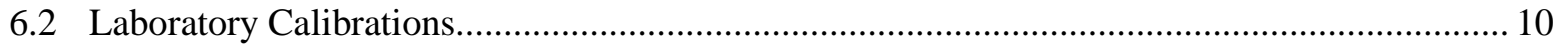

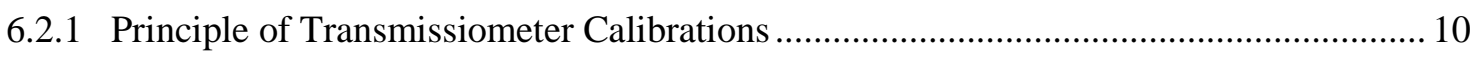

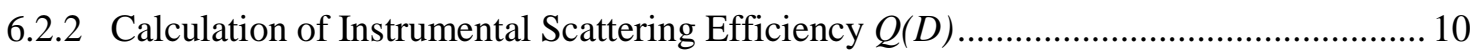

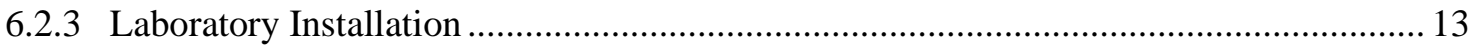

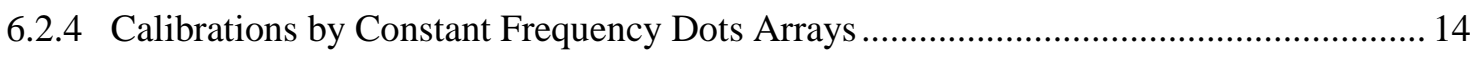

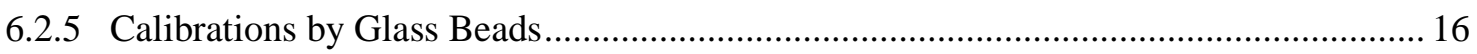

6.2.6 Calibrations by Constant Frequency Stellar Grids ...................................................... 21

6.2.7 Calibrations by Irregular-shaped Particles .................................................................. 22

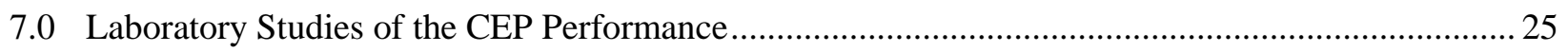

7.1 Effect of the Beam Inhomogeneity ……........................................................................ 25

7.2 Temperature Effect on the CEP Performance ................................................................... 26

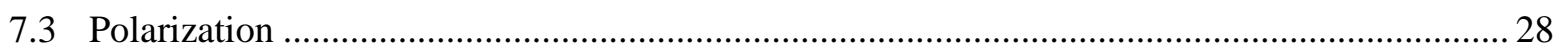

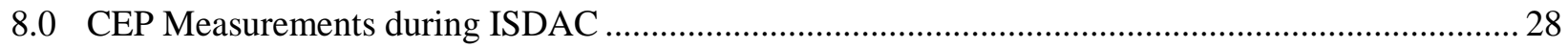

8.1 Calculations of the Extinction Coefficient from FSSP and CDP Measurements..................... 29

8.2 Calculations of the Extinction Coefficient from Imaging Probes ........................................... 29

8.3 Comparisons of the CEP and Particle Probe Data in Liquid Clouds ...................................... 30

8.4 Comparisons of the CEP And Particle Probe Data in Ice ..................................................... 32

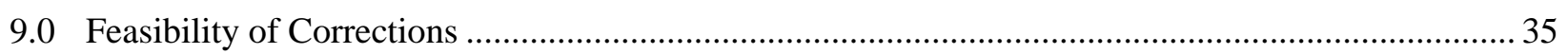

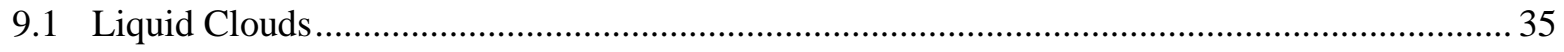

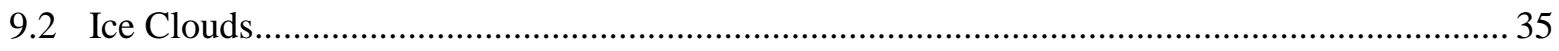

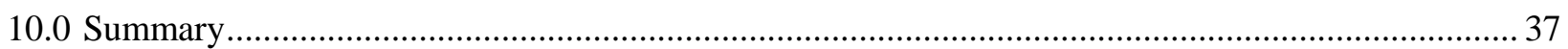

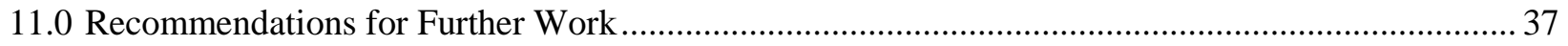

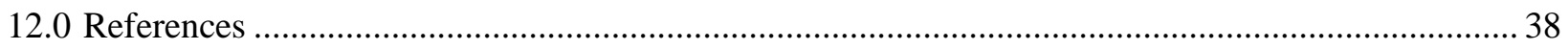




\section{Figures}

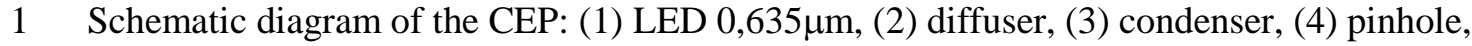
(5) objective, (6) cone cube retroreflector, (7) beam-splitter, (8) photodetector, (9) optical chopper, (10) optocouple, (11) bandpass filter, and (12) front heated glass........................................ 4

2 Cloud Extinction Probe: optical unit (top), control box (bottom), and retroreflector (right)............... 4

3 Installation of the Cloud Extinction Probe on the NRC Convair 580 ............................................. 5

4 A collimated stream of photon packets is emitted from the source/receiver lens toward the retroreflector

5 Extinction correction values 1- $f$, see (10), as determined by Monte Carlo simulation (heavy lines) and (12) (light lines) for water spheres and various pin-hole aperture sizes (as listed on

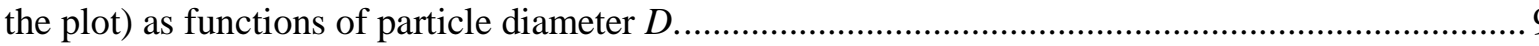

6 Scheme explaining calculation of the extinction efficiency from laboratory measurements for the cases of (a) one target and (b) two targets.

7 Scheme explaining calculation of the effect of the substrate of the attenuation of light by the

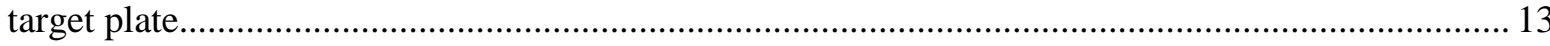

8 Laboratory installation used for the calibrations of the Cloud Extinction Probe.............................. 14

9 Dot arrays used for calibration of the Cloud Extinction Probe...................................................... 15

10. Custom-made calibrating dot grids with different dot diameters ................................................... 15

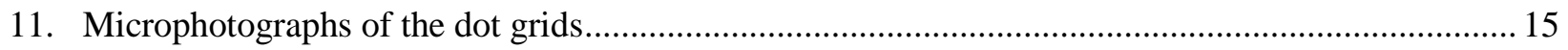

12. Instrumental extinction efficiency obtained from the CEP calibration by the dot arrays. Black solid curves are theoretical calculations of $Q(D)$ for water spheres for different receiving aperture angles (section 2.1)........................................................................................ 16

13 Examples of images of glass beads in the microscope's field of view........................................... 17

14 Explanation of different effects causing errors in sizing of images of particles:

(a) inhomogeneity of background intensity, (b) different threshold intensity in conversion of grayscale into b/w image, (c) aberration of distortion.

15 Contribution of different corrections on the reconstructed size distribution deduced from the analysis of the image frame of the $125 \mu \mathrm{m}$ dot grid. (a) Corrections on the inhomogeneity of background intensity, (b) effect of the threshold intensity on image sizes, (c) effect of the distortion aberration corrections.

16. Determination of the cut-off level for conversion of a grayscale image into black-and-white for subsequent particle sizing and determination of particle area coverage.

17 Sequence of procedures during image processing and calculation of $\mathrm{S} / \mathrm{S}_{0}$

18. Instrumental extinction efficiency versus diameter calculated from the CEP calibrations for monodisperse glass beads.

19 Arrangement of the stellar patterns on the "stellar" grids.......................................................... 21

20 Visual appearance of the stellar grids under microscope.

21. Instrumental scattering efficiency obtained from the CEP calibrations by stellar arrays with three different sizes: $L=500 \mu \mathrm{m}, 1000 \mu \mathrm{m}$, and $2000 \mu \mathrm{m}$. 
22. Microphotographs of sugar crystals (left) and corresponding distributions of $X, Y$, and $D_{\text {eff }}$ sizes (right).

23. Microphotographs of broke glass particles (left) and corresponding distributions of $X, Y$, and $D_{\text {eff }}$ sizes (right).

24. Instrumental scattering efficiency obtained from the CEP calibrations for sugar crystals and broken glass particles as shown in Figures 22 and 23.

25. CEP output signal versus position along the beam direction of the $15-\mu \mathrm{m}$ (a) and $2000-\mu \mathrm{m}$

(b) dot arrays. 25

26 CEP output signal versus position of the retroreflector across the beam.......

27. Experimental setup for the temperature sensitivity tests in the cold room: (1) CEP, (2) optical window, (3) retroreflector, (4) condenser lens, (5ab) bandpass filter, and (6) optical spectrophotometer.

28 Pictures of the elements of the experimental setup shown in Figure 27........................................ 26

29 Spectral transmittance of the bandpass filter installed in the CEP. .................................................2 27

30 Spectral irradiance of the LED measured at different temperatures............................................... 27

31. LED spectrum width (a), wavelength corresponding to the max irradiance in the LED spectrum (b), LED max irradiance (c) deduced from the data shown in Figure 30........................... 28

32 Polarization sensitivity of the CEP in (a) polar and (b) Cartesian coordinates.................................. 28

33 Conceptual diagram of calculation of extinction coefficient from OAP-2D imagery........................ 30

34. Time series of the measurements of extinction coefficient by the CEP and particle probes FSSP and CDP (a), and Rosemount Icing Detector signal (b).

35. Scatter diagrams of extinction coefficient measured by CEP and particle probes FSSP (a) and CDP (b) in liquid clouds on 26 April 2008 during the ISDAC field campaign (Figure 34).

36. Distribution of extinction coefficient measured by FSSP and averaged over the time period corresponding to that shown in Figure 34 (a).

37. Time series of the measurements of extinction coefficient by the CEP and particle probes OAP-2DC and OAP-2DP (a) and Rosemount Icing Detector signal (b).

38. Scatter diagrams of extinction coefficient measured by CEP and OAP-2DC (a) and particle images measured by OAP-2DC (b) for the time interval shown in Figure 37.

39. Distribution of extinction coefficient calculated from OAP-2DC and averaged over the time period corresponding to that shown in Figure 37 (a)

40. Time series of the measurements of the extinction coefficient by the CEP and particle probes OAP-2DC and OAP-2DP (a) and Rosemount Icing Detector signal (b).

41. Scatter diagrams of extinction coefficient measured by the CEP and OAP-2DC (a) and OAP2DP (b) during the flight shown in Figure 40

42. Distribution of extinction coefficient calculated from OAP-2DC/2DP and averaged over the time period corresponding to that shown in Figure 40.

43 CPI imagery of ice particles.

44. Image of a stellar ice particle showing multiple small-scale features in its internal structure. 


\section{Acronyms and Abbreviations}

2D

CDP

CEP

DAC

EC

ERB

FSSP

ISDAC

NRC

OAP

RICE

STAC two-dimensional

Cloud Droplet Probe

Cloud Extinction Probe

direct area calculation

Environment Canada

Earth's radiation budget

Forward Scattering Spectrometer Probe

Indirect and Semi-Direct Aerosol Campaign

National Research Council

Optical Array Probe

Rosemount Ice Detector

size-to-area conversion 


\subsection{Introduction}

Extinction coefficient is one of the fundamental microphysical parameters characterizing bulk properties of clouds. Knowledge of extinction coefficient is of crucial importance for radiative transfer calculations in weather prediction and climate models given that Earth's radiation budget (ERB) is modulated much by clouds. In order for a large-scale model to properly account for ERB and perturbations to it, it must ultimately be able to simulate cloud extinction coefficient well. In turn this requires adequate and simultaneous simulation of profiles of cloud water content and particle habit and size. Similarly, remote inference of cloud properties requires assumptions to be made about cloud phase and associated singlescattering properties, of which extinction coefficient is crucial. Hence, extinction coefficient plays an important role in both application and validation of methods for remote inference of cloud properties from data obtained from both satellite and surface sensors (e.g., Barker et al. 2008).

While estimation of extinction coefficient within large-scale models is relatively straightforward for pure water droplets, thanks to Mie theory, mixed-phase and ice clouds still present problems. This is because of the myriad forms and sizes that crystals can achieve, each having their own unique extinction properties. For the foreseeable future, large-scale models will have to be content with diagnostic parametrization of crystal size and type. However, before they are able to provide satisfactory values needed for calculation of radiative transfer, they require the intermediate step of assigning singlescattering properties to particles. The most basic of these is extinction coefficient, yet it is rarely measured directly, and therefore verification of parametrizations is difficult. The obvious solution is to be able to measure microphysical properties and extinction at the same time and for the same volume. This is best done by in situ sampling by instruments mounted on either balloon or aircraft. The latter is the usual route and the one employed here. Yet the problem of actually measuring extinction coefficient directly for arbitrarily complicated particles still remains unsolved.

The following subsections document the history of attempts to directly measure cloud extinction, the current measurement device known as the Cloud Extinction Probe (CEP), specific problems with direct measurement of extinction coefficient, and the attempts made here to address these problems.

\subsection{Background}

The extinction process of a dispersed medium consisting of cloud particles suspended at random in the air can be described by the Beer-Bouguer law as

$$
I=I_{0} e^{-\beta(\lambda) L}
$$

where $I_{0}$ is incident light intensity, $I$ is light intensity transmitted through the medium in the forward direction (i.e., parallel to the incident light), $\beta(\lambda)$ is volumetric extinction coefficient, and $L$ is geometric distance between emitter and receiver.

Early attempts to use airborne extinctiometers for measurements of visibility in clouds go back to work by Kampe (1950) and Weickmann and Kampe (1953). The first airborne extinctiometer utilized the transmissiometric method. It consisted of an incandescent lamp, a collimator, and a photocell for 
measuring light intensity. The source of light and the photocell were mounted on the wing and separated by a few metres. Zabrodsky (1957) built an airborne double-pass transmissiometer where light travelled to a retroreflector and back, where it was then measured by a photodetector. Nevzorov and Shugaev (1972, 1974) built an advanced version of the double-pass transmissiometer with improved stability and higher sensitivity. This was a successful design that allowed for the collection of a large data set of extinction coefficient for different types of clouds (Kosarev et al. 1976, Korolev et al. 2001). King and Handsworth (1979) built a single-pass transmissiometer with an ultraviolet source of light generated by a germicidal lamp. Zmarzly and Lawson (2000) designed a multi-pass and multi-wavelength Cloud Extinctiometer. Gerber et al (2000) constructed a Cloud Integrated Nephelometer where the extinction coefficient was calculated from an arrangement of four Lambertian sensors, two of which had cosine masks.

In many studies cloud extinction coefficient was estimated from composite size distributions measured by several cloud spectrometers. Earlier measurements (Korolev et al. 1999) showed good agreement between extinction coefficient measured by a cloud transmissiometer and that derived from the Particle Measuring Systems Forward Scattering Spectrometer Probe (FSSP) droplet size spectra. However, calculation of extinction coefficient from particle size distributions in ice and mixed-phase clouds is subject to potentially large errors due to uncertainties related to the size-to-area conversion technique, shattering issues, and limited accuracy in measurements of concentration and sizes of ice particles smaller than approximately $100 \mu \mathrm{m}$.

Despite the great influence of extinction coefficient on our ability to simulate radiation transfer for cloudy atmospheres and Earth's climate in general, probes that are capable of direct measurement of extinction coefficient have not become a part of conventional airborne microphysical instrumentation. The effort to fill this gap has been undertaken by Cloud Physics Research and the Severe Weather Section of Environment Canada (EC). The CEP was designed and built in 2006 by EC for airborne measurement of cloud extinction coefficient (Korolev 2008) and has since operated successfully during several flight campaigns.

\subsection{Principles of Operations and Description of CEP}

The CEP utilizes the transmissiometric method. The principle of operation is based on having emission of visible light of known strength followed by its measurement after having traversed and been attenuated by a known volume. This method enables calculation of extinction coefficient from first principles based on Eq.1. The CEP consists of an optical unit that combines a transmitter and receiver as well as a retroreflector. Figure 1 shows a general schematic of the optical unit. A collimated light beam is generated by an optical system consisting of a super-bright LED at wavelength $\lambda=0.635 \mu \mathrm{m}$ (1), diffuser (2), condenser (3), pinhole (4), and objective (5). The beam travels from the optical unit to the retroreflector (6), and then returns the same distance back to the optical unit. Then, after passing though the objective and beam-splitter (7), its intensity is measured by a photodetector (8). The optical chopper (9) modulates the light beam and controls, turning the LED on and off with the help of the optocouple (10). The optical chopper consists of a sequence of holes, dark areas, and mirrors glued on its surface. During the first half of the period when the hole is opened, the LED is on, and the photodetector measures the intensity of transmitted light plus the background intensity $\left(I_{t o t}\right)$. During the second half of the period when the hole is opened, the LED is off, and the photodetector measures the intensity of the background 
light $\left(I_{b k g}\right)$. During the first half of the period when the hole is closed and the LED on, light is reflected from the mirrored surface. After passing through a beam-splitter, the reflected light is measured by the photodetector $\left(I_{\text {norm }}\right)$. This signal characterizes the intensity of the LED and is used to normalize all other measured signals. During the second half of the period when the chopper hole is closed, the LED is on and the beam hits the blackened surface of the chopper. In this case, the photodetector measures the signal $\left(I_{i n t}\right)$ related to the light scattered inside the optical unit due to reflection from the optical surfaces and the different parts inside the probe's housing. The advantage of such a scheme is that it allows measurements of the intensities of the LED, background, and attenuated light with the same photodetector. Utilizing the above scheme minimizes the effect of changes of the photodetector sensitivity during flight (e.g., caused by temperature drift) on the measurements of the extinction coefficient.

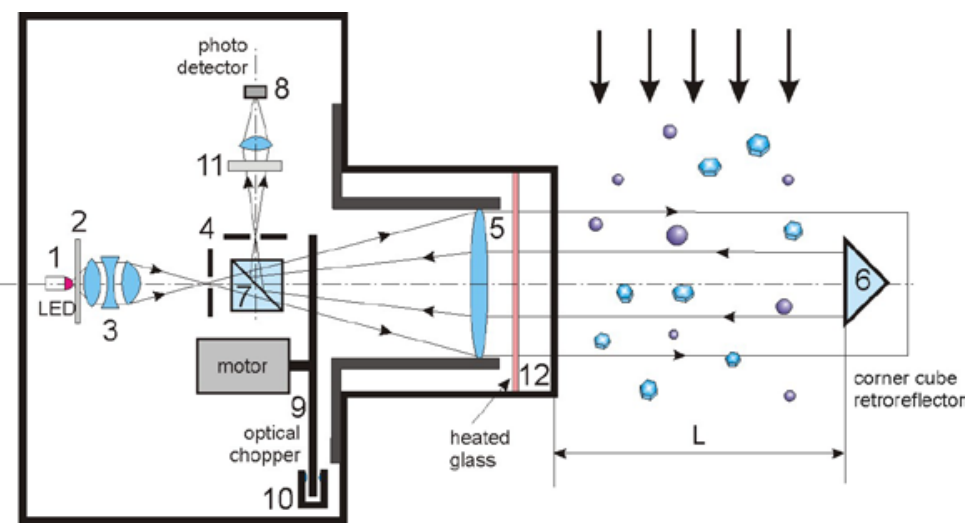

Figure 1. Schematic diagram of the CEP: (1) LED 0,635 $\mu \mathrm{m}$, (2) diffuser, (3) condenser, (4) pinhole, (5) objective, (6) cone cube retroreflector, (7) beam-splitter, (8) photodetector, (9) optical chopper, (10) optocouple, (11) bandpass filter, and (12) front heated glass.

The optical scheme was designed to produce a highly uniform collimated beam. The inhomogeneity of the light intensity across the beam does not exceed $2 \%$. This minimizes the effect of vibration and mutual motion by the optical unit and retroreflector with respect to each other during the flight operation. A similar approach has been used by Nevzorov and Shugaev (1974). The size of the retroreflector was chosen so that its displacement from the center of the beam in each direction at the distance of approximately $1 \mathrm{~cm}$ does not affect the output signal.

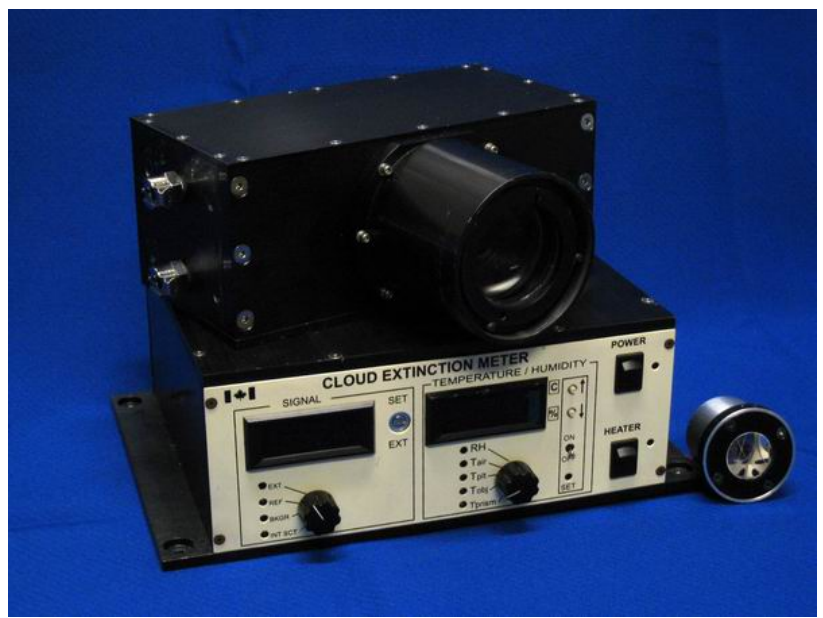

Figure 2. Cloud Extinction Probe: optical unit (top), control box (bottom), and retroreflector (right). 
During the flight the retroreflector always stayed inside the beam within the light intensity homogeneous area, whereas the reflected beam always stayed within the front lens area. This provides a stable output signal with a reduced sensitivity to the aircraft vibration.

The CEP was designed to operate in all weather conditions. The optics of the probe are well heated to prevent fogging during rapid aircraft descent at vertical speeds greater than $5 \mathrm{~m} / \mathrm{s}$. The environment inside the optical unit is temperature-controlled so that the instrument can operate at air temperatures as low as $60^{\circ} \mathrm{C}$. Based on the flight tests, the threshold sensitivity of the probe was found to be approximately $0.2 \mathrm{~km}^{-1}$. The upper limit of the measured extinction coefficient is estimated to be no less than $200 \mathrm{~km}^{-1}$.

The CEP was installed on the National Research Council (NRC) Convair 580. The optical unit was mounted inside the wing-tip canister and the retroreflector inside a hemispherical cap at the rear side of a PMS probe canister (Figure 3). Distance between the optical unit and retroreflector was $L=2.35 \mathrm{~m}$.

The sample area of the probe is defined by the length of the beam $(L)$ and the diameter of the reflector ( $d=25 \mathrm{~mm}$ ) and is calculated as $S=L d$. For the Convair-580 installation, $S \approx 0.06 \mathrm{~m}^{2}$. At a typical airspeed of $100 \mathrm{~m} / \mathrm{s}$, the corresponding cloud volume sampling rate is approximately $6 \mathrm{~m}^{3} / \mathrm{s}$. Assuming a decent sensitivity, the above sampling rate allows measurements of a statistically significant extinction coefficient of ice particles with concentration of a few per cubic meter.

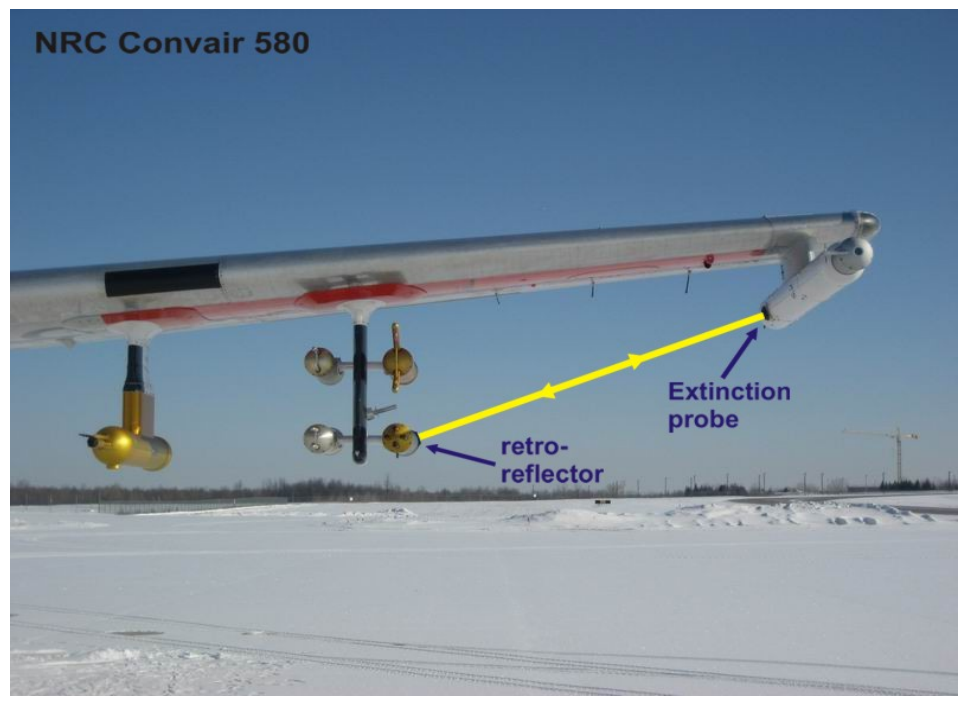

Figure 3. Installation of the Cloud Extinction Probe on the NRC Convair 580. The yellow line indicates the position of the beam under the wing.

\subsection{Forward Scattering}

Beginning in the early 1950s, it was recognized that the transmissiometric method is prone to contamination by photons that had been scattered into the near-forward direction passing through the receiving aperture. Hence, as long as one interprets the measured intensity of light as though it were unattenuated, extinction coefficient will be underestimated systematically through inversion of Eq. 1 . Theoretical considerations (Gumprecht and Sliepevich 1953, Deepak and Box 1978) showed that the 
effect of forward scattering on the measured extinction coefficient depends on the angle of the receiving aperture $(\varphi)$ and the scattering phase function of particles. Mie scattering calculations show that forward scattering is relatively weak for small particles, and so in the case of small droplets, underestimation of extinction coefficient will be small. Large particles, however, have strong forward-scattering lobes that may result in significant underestimation of measured extinction coefficient. Theoretical calculations show that for large particles, the relative error in extinction coefficient measurements approaches a factor of two.

\subsection{Objectives}

Until now, due to the absence of calibrating standards, there have been no experimental techniques capable of estimating the errors in extinction coefficient measurements versus particle sizes. Gumprecht and Sliepevich (1953) attempted to utilize a set of monodisperse glass beads suspended in water to measure forward scattering. However, the accuracy of maintaining the constant bulk number concentration in the test cell was low. The number concentration is also affected by the rate of sedimentation for large glass beads. Environment Canada developed a technique and designed a laboratory installation for the CEP calibration. The main objective of this calibration is to obtain a calibration curve of the extinction efficiency $Q$ versus particle size $D$, i.e., $Q(D)$. Due to the theoretical analysis (Deepak and Box 1978) the value of $Q(D)$ is supposed to vary between 2 for small particles and 1 for large particles. Knowledge of the function $Q(D)$ would allow one to make corrections to measured extinction coefficient when a particle size distribution is measured simultaneously with the CEP extinction coefficient.

Below is a description of the work to be performed within the frame of this project:

1. Design and fabricate a laboratory installation for the calibration of the CEP.

2. Perform laboratory calibrations of the CEP with the help of constant frequency dot arrays and glass beads. Based on the results of the calibrations, determine corrections to measured extinction coefficient versus particle size.

3. Perform a theoretical analysis of the corrections to extinction coefficient.

4. Apply corrections deduced from laboratory calibrations to the CEP data set collected during the ISDAC flight campaign. Develop parameterization of extinction coefficient measured in ice clouds versus temperature.

5. Determine the effect of forward scattering on the extinction coefficient measurements

\subsection{Effect of Forward Scattering on the Extinction Coefficient Measurements}

\subsection{Theoretical Calculations of the Effect of Forward Scattering}

In an attempt to better comprehend the nature of the observations, we constructed a numerical model of the extinctiometer. Rather than attempt to build an analytic approximation, it was decided that a Monte 
Carlo photon transfer-based solution would better serve our purposes in light of the complicated character of ice scattering phase functions. This subsection describes briefly the Monte Carlo technique.

To begin, $0.635 \mu \mathrm{m}$ wavelength photon packets are emitted uniformly from the source lens, travelling perpendicular to the lens toward the retroreflector. Distance to the first scattering event is computed as

$$
d_{s}=-\frac{\ln R}{\beta}
$$

where $R$ is a uniform random number between 0 and 1 , and $\beta$ is extinction coefficient $\left(\mathrm{km}^{-1}\right)$. Scattering angle is determined by first solving for $\theta_{\mathrm{s}}$ as

$$
R=\frac{1}{2} \int_{0}^{\theta_{s}} p^{\prime}(\theta) \sin \theta d \theta
$$

where $p^{\prime}(\theta)$ is the azimuthally averaged scattering phase function in which

$$
\frac{1}{2} \int_{0}^{\pi} p^{\prime}(\theta) \sin \theta d \theta=1
$$

This gives the deflection angle $\theta_{\mathrm{s}}$ away from the incident ray. Then one solves for the azimuthal angle $\varphi_{\mathrm{s}}$ as

$$
R=\frac{\int_{0}^{\varphi_{s}} p\left(\theta_{s}, \varphi\right) d \varphi}{\int_{0}^{2 \pi} p\left(\theta_{s}, \varphi\right) d \varphi},
$$

where $p\left(\theta_{\mathrm{s}}, \varphi\right)$ is the full phase function given $\theta_{\mathrm{s}}$. If phase functions are azimuthally symmetric, as they are for spherical water droplets, (5) simplifies to

$$
R=2 \pi \varphi,
$$

For this version of the model, aerosols and Rayleigh scatter are neglected. Moreover, it is assumed that particles can be represented as point scatterers and that $p(\theta, \varphi)$ and $p^{\prime}(\theta)$ can be represented by far-field solutions (e.g., Mie theory for homogeneous spherical particles). This is a valid assumption given that cloud particles are generally expected to be small compared to most distances between scattering events and receiver (Mishchenko et al. 2006).

The possible fates of a photon are shown in Figure 4. If a scattered photon strays outside the emitted column of light by more than 5 times the radius of the lens $d$, it is considered to have exited the experiment. Note that detectable photons are not limited simply to those that are transmitted directly or undergo just a single forward-scattering event (e.g., Deepak and Box 1978). Photons that undergo multiple scattering events, including backscatter, are also detected. However, these are generally very minor contributions for values of $\beta$ within the extinctiometer's reliable operating range. Hence, to a very good approximation, the extinctiometer is effectively a single-pass device with separate source and receiver. Nevertheless, this Monte Carlo routine is fast, provides useful benchmarks, and, importantly, 
like most Monte Carlo treatments, can accommodate arbitrarily complicated, non-analytic scattering phase functions.

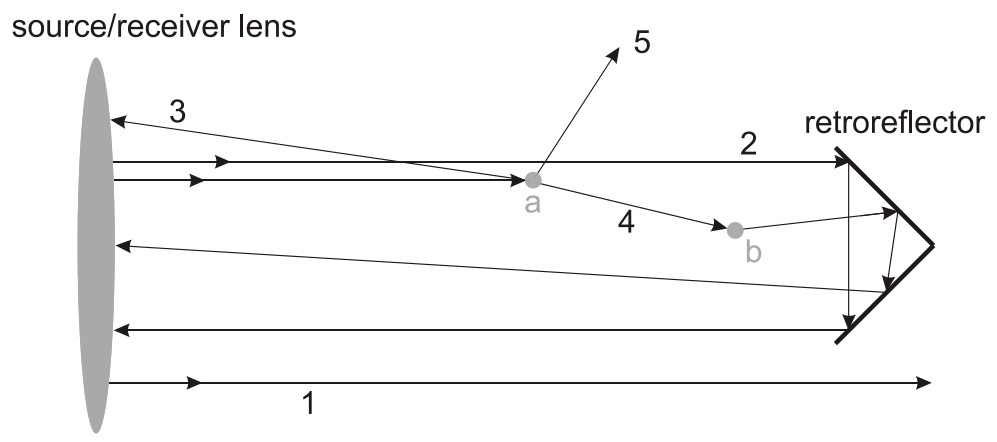

Figure 4. A collimated stream of photon packets is emitted from the source/receiver lens toward the retroreflector. The fate of a packet can be: (1) emitted directly, or scattered, past the retroreflector and out of the experiment (all photons scattered behind the retroreflector are considered to be out of the experiment); (2) transmitted unattenuated to the retroreflector and back unattenuated to the receiver (true direct-beam transmittance); (3) scattered one or more times by particles into the receiver without interacting with the retroreflector; (4) scattered one or more times by particles into the retroreflector and back into the receiver (including scattering after encountering the retroreflector); (5) scattered by a particle, or the retroreflector, out of the experiment.

Let $N_{T}$ and $N_{R}\left(\theta^{*}\right)$ be the numbers of photons emitted out and received at the lens of diameter $d$, respectively. In order for a photon to be received (i.e., detected), its angle of incidence relative to normal to the receiver's aperture has to be less than $\theta^{*}$. For the experiments performed here, transmittance along the path from the source to the retroreflector, whose radius is $r$, and back is defined as

$$
T^{\prime}\left(\theta^{*}\right)=\frac{N_{R}}{N_{T}\left(\frac{r}{d}\right)^{2}},
$$

Assuming Beer's Law for a collimated beam of light, and particles distributed entirely at random, the transmitted fraction of unattenuated photons is given by

$$
T=\exp (-2 L \beta)
$$

where $L$ is distance $(\mathrm{km})$ from source to retroreflector. However, because the receiver cannot discriminate scattered from completely unattenuated photons, if one tries to force Beer's Law onto (7), the implication is that

$$
\beta(\theta *)=-\frac{1}{2 L} \ln \left(\frac{N_{R}}{N_{T}\left(\frac{r}{d}\right)^{2}}\right),
$$


which underestimates true extinction $\beta$, primarily because of single forward-scattering events. Hence, by defining

$$
T^{\prime}\left(\theta^{*}\right)=\exp \left\{-2 L \beta\left[1-f\left(\theta^{*}\right)\right]\right\}
$$

where $\mathrm{f}\left(\theta^{*}\right)$ represents all contributions other than unattenuated direct-beam, the problem comes down to finding

$$
f\left(\theta^{*}\right)=\frac{\beta\left(\theta^{*}\right)}{\beta},
$$

In general, $\mathrm{f}\left(\theta^{*}\right)$ will also depend on particle size $\mathrm{D}$ and type. If one assumes that single-scattering prevails and that the portion of the phase function that leads to scattered photons being detected does not depend on the location of the scattering event (i.e., small $\theta^{*}$ ), and that backscattered photons are of no consequence, a very good approximation is obtained by

$$
f\left(\theta^{*}\right)=\frac{1}{2} \int_{0}^{\theta^{*}} p^{\prime}(\theta ; r) \sin \theta d \theta
$$

If these conditions are violated, or if scattering properties depend much on particle orientation, (12) could lead to untenable errors. Figure 5 shows, however, that for water spheres at small $\theta^{*}$ (i.e., small pin-hole apertures) and $D$ less than about $500 \mu \mathrm{m}$ (12) is extremely accurate.

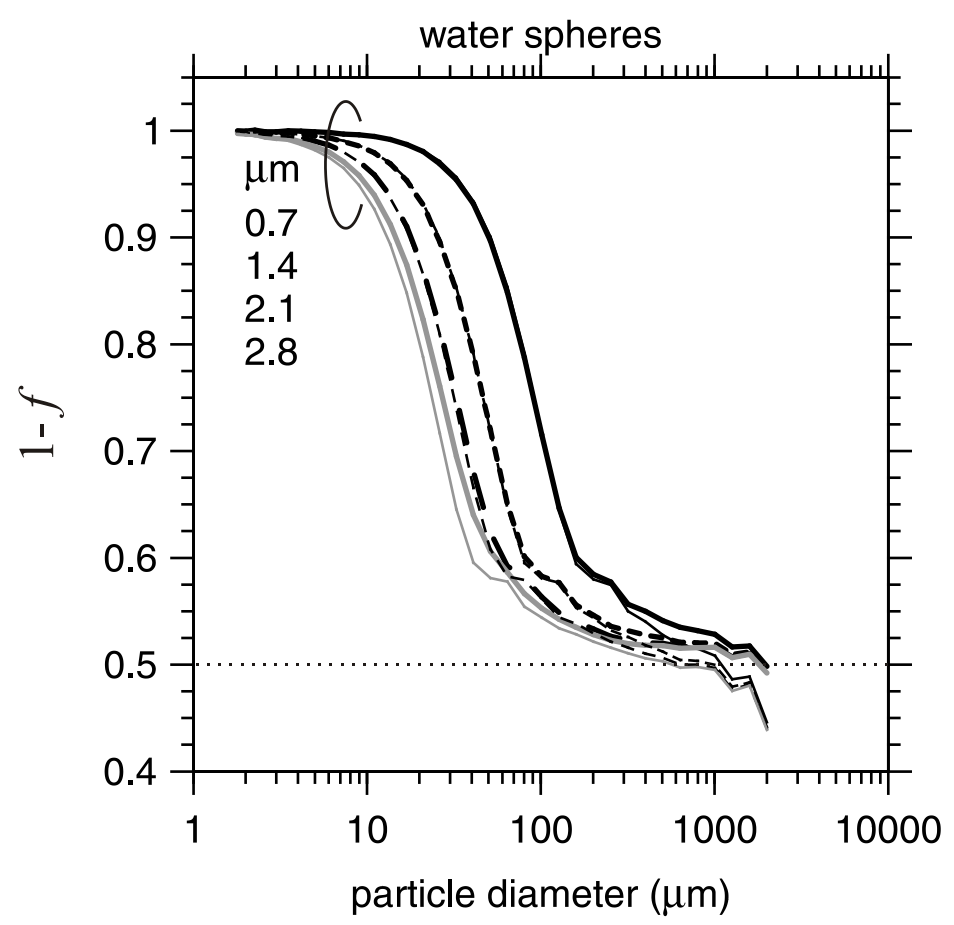

Figure 5. Extinction correction values 1- $f$, see (10), as determined by Monte Carlo simulation (heavy lines) and (12) (light lines) for water spheres and various pin-hole aperture sizes (as listed on the plot) as functions of particle diameter $D$. 


\subsection{Laboratory Calibrations}

\subsubsection{Principle of Transmissiometer Calibrations}

The essence of calibration of transmissiometers consists in transmitting the probes' beam through a media with a known particle dispersion and extinction coefficient and then performing subsequent comparisons between the measured and known extinction coefficients. Such calibrations are hindered by the absence of calibrating standards. The main challenge in the calibrations is developing an environment with controlled particle size distribution and extinction coefficient. Until now there have been no known procedures for calibration of transmissiometers and for obtaining $Q(D)$.

In order to fill this gap, Environment Canada has developed a technique for the estimation of $Q(D)$ from laboratory measurements. The principle of the calibration consists in passing the beam through a flat optical target with a known attenuating pattern. The extinction coefficient of the target is calculated from the attenuating pattern (e.g., Figure 9) and then compared with the measured one.

For the calibration purposes several types of targets have been used. The first types of targets consisted of a set of glass plates coated with a repeating pattern of (a) equally spaced opaque dots with known diameters $D$, and (b) six-ended stars simulating stellar ice crystals. Such optical targets have predetermined area coverage (or area ratio) $S / S_{0}$, where $S$ is total area of opaque dots covering area $S_{0}$. The intensity of the incident light $I_{0}$ and that passed through the target $I$ are related as

$$
\frac{I_{0}-I}{I_{0}}=Q(D) \frac{S}{S_{0}}
$$

Such a technique enables estimation of $Q$ for each individual target. Details of calculations of the function $Q(D)$ are described in Section 6.2.2.

The second types of optical targets were monodisperse glass beads randomly spread over the surface of a glass substrate. Calculation of the area ratio $S / S_{0}$ was deduced from the processing of microphotographs of the beads.

The third types of targets were particles with random shapes such as crystals of sugar or broken glass.

The results of the CEP calibrations by three types of targets are described in Sections 6.2.4-6.2.7.

\subsubsection{Calculation of Instrumental Scattering Efficiency $Q(D)$}

Consider the Beer law in the differential form for a medium consisting of monodisperse particles with the concentration $n$ and thickness $d z$

$$
d I=\sigma n I_{0} d z
$$

where $d I=I_{1}-I_{0}$ is the changes of the intensity of the light beam after passing through a medium; $\sigma=Q s$ is the scattering cross-section of one particle; $s$ is the geometric cross-section of the particle; and 
$Q$ is the extinction efficiency. The particle concentration $n$ can be presented as $n=N / V=N / S_{0} d z$, where $N$ is the number of particles in the volume $V$ and $S_{0}$ is the cross section of the beam. Then substituting $n$ and $\sigma$ in Eq.14 yields

$$
\frac{I_{0}-I_{1}}{I_{0}}=Q \frac{S}{S_{0}}
$$

Here, $S=N s$ is the total geometrical area of the particles.

For the double-pass scheme (Figure 6a) light passes twice (forward and back) through the target. So, after the second pass, the intensities of the incident and transmitted light are related as

$$
\frac{I_{1}-I}{I_{1}}=Q \frac{S}{S_{0}}
$$

Combining Eqs. 15 and 16 and excluding $I_{1}$ yields

$$
Q=\frac{1-\sqrt{\frac{I}{I_{0}}}}{\frac{S}{S_{0}}}
$$

During the calibrations, two spatially separated targets (Figure 6b) were also used. For the case of two targets with the same dots, it can be shown that the intensities $I / I_{0}$ and area rations $S / S_{0}$ are related as

$$
Q=\frac{1-\left(\frac{I}{I_{0}}\right)^{1 / 4}}{\frac{S}{S_{0}}}
$$

For the case of $k$ targets $I / I_{0}$ and $S / S_{0}$ are related as

$$
Q=\frac{1-\left(\frac{I}{I_{0}}\right)^{1 / 2 k}}{\frac{S}{S_{0}}}
$$




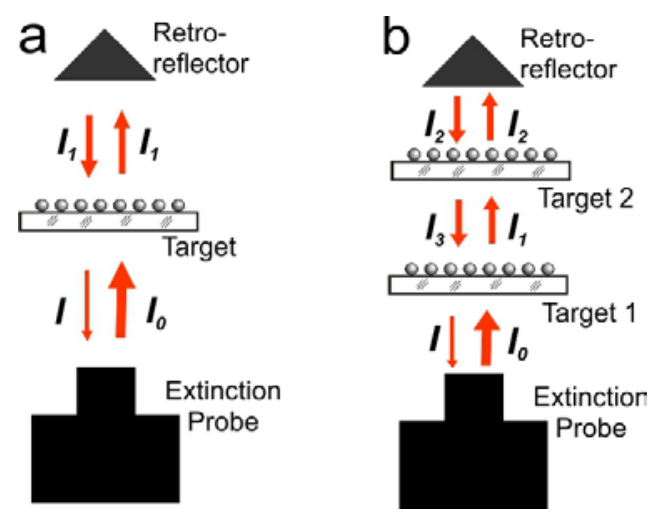

Figure 6. Scheme explaining calculation of the extinction efficiency from laboratory measurements for the cases of (a) one target and (b) two targets.

Eq.17 considers an ideal case, assuming that the substrate is absolutely transparent and does not attenuate light. In fact, the substrate reflects light in the backward direction, and the material it is made of may absorb some fraction of light. This will result in a systematic bias in the measurements of the attenuation of the light. This effect was accounted for with the help of special transparent plates made of the same material as the target plates, but without dot arrays on their surfaces. In every session of measurements of the signal attenuation by the dot arrays, the beam attenuation by a blank glass plate was measured as well in order to estimate the effect of the substrate.

Figure 7 provides an explanation for the calculations of the effect of the substrate. In order to estimate the effect of the substrate, the target plate was considered as consisting of a "pure" coating and the glass substrate (Figure 7a). The intensities of light transmitted through this system can be described by the following system of equations

$$
\begin{gathered}
\frac{I_{0}-I_{1}}{I_{0}}=A \\
\frac{I_{1}-I_{2}}{I_{1}}=Q \frac{S}{S_{0}} \\
\frac{I_{2}-I_{3}}{I_{2}}=Q \frac{S}{S_{0}} \\
\frac{I_{3}-I}{I_{3}}=A
\end{gathered}
$$

The effect of a substrate (Figure 7b) can be written as

$$
\frac{I_{0}-I_{1}}{I_{0}}=A
$$




$$
\frac{I_{1}-I_{\text {glass }}}{I_{1}}=A
$$

Solving the system Eqs. (20)-(25) yields

$$
Q=\frac{1-\sqrt{\frac{I}{I_{\text {glass }}}}}{\frac{S}{S_{0}}}
$$

Eq.26 is similar to Eq.17, with the only difference that $I_{o}$ in Eq.17 is replaced by $I_{\text {glass }}$.

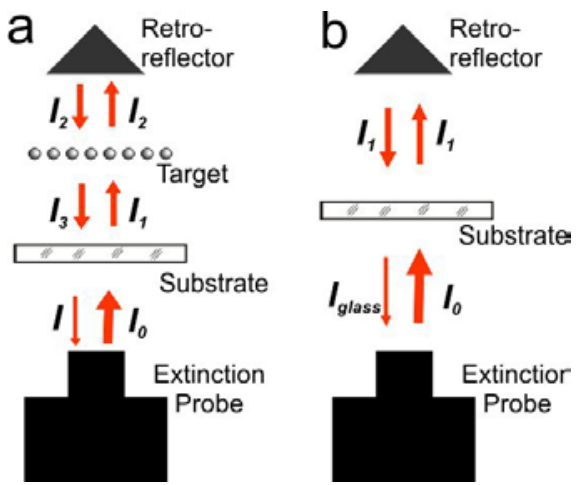

Figure 7. Scheme explaining calculation of the effect of the substrate of the attenuation of light by the target plate.

The values of $I, I_{\text {glass }}, S / S_{0}$ can be obtained from the measurements and thus yield estimates of $Q$ from Eq.26. The physical meaning of $Q$ calculated from Eq.26 should be interpreted as "instrumental” extinction efficiency. The instrumental extinction efficiency primarily depends on the receiving aperture of the transmissiometer. The objective of the laboratory studies described below is to determine $Q(D)$ and to estimate the feasibility of corrections depending on the type of cloud particles (ice or liquid) and their dispersion.

\subsubsection{Laboratory Installation}

To achieve the calibration goals, the following installation was designed and built in the laboratory facility of the Cloud Physics and Severe Weather Research Section. The picture of the laboratory installation is shown in Figure 8. The laboratory installation consisted of a vertically oriented frame with the CEP optical unit mounted on one end and a retroreflector on the other. The distance between the CEP and the retroreflector was the same as that on the NRC Convair $580(2.30 \mathrm{~m})$. The optical targets were installed on a horizontal platform. The platform with the target was mounted on a set of rails, which enabled positioning of the targets in a vertical direction between the CEP and the retroreflector. The microphotography of the optical targets (e.g., glass beads) was conducted with the help of a highresolution CCD camera (Lumenera X32) attached to a microscope. The CCD matrix of the camera has 1216 x 1616 elements. However, micro-shifting technology utilized in this camera allowed images with a linear resolution four times higher, resulting in images with 4864 x 6464 pixels. The microscope used in 
these calibrations has a long working distance varying from $13 \mathrm{~mm}$ to $89 \mathrm{~mm}$, depending on the optical magnification. A set of high-quality changeable Mitutoyo optics and zoom options provided highresolution imagery of micro-objects ranging in size from a few microns to a few millimetres. The microscope was mounted on a three-positioning stage, which allowed it to be moved in and out of the CEP beam. As a result, it was possible to take microphotographs of the target and to measure light attenuation by the CEP without touching the optical target. This is important when working with glass beads, which can easily start moving over the substrate surface in case of minor vibrations.

Proper illumination is one of the critical components for obtaining high-quality microscope imagery. A rectangular 10 x $10 \mathrm{~cm}$ green LED array backlight (CCS Inc, Kyoto, Japan) provided a uniform diffuse illumination of the targets, without leaving hot spots. The backlight illuminator was mounted on a rail with a slider-lock, which could be positioned vertically in order to achieve the best quality of particle imagery depending on the microscope optics and magnification. The backlight illuminator holder design created the possibility of moving the illuminator in and out the CEP beam, in order to take microphotographs of the optical targets and measurements of the light intensity without any significant rearrangement of the installation.

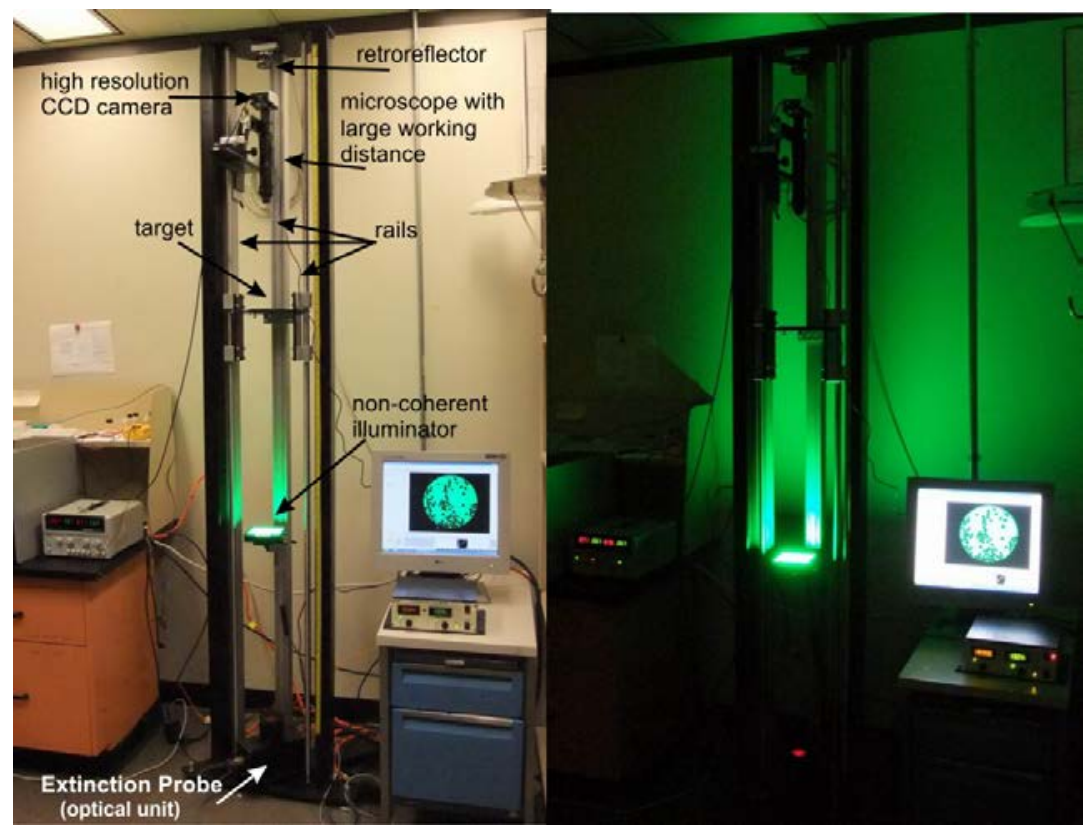

Figure 8. Laboratory installation used for the calibrations of the Cloud Extinction Probe.

\subsubsection{Calibrations by Constant Frequency Dots Arrays}

A set of custom-made constant frequency dot arrays were manufactured (Applied Optics Inc.) for the calibrations of the CEP. The constant frequency arrays consist of 1-mm-thick glass plates coated with equally spaced blue chrome opaque dots with diameter $D$ (Figure 9). The glass plates had an antiglare coating to mitigate reflection and increase transmittance. The distance between the centers of the dots in $\mathrm{X}$ - and $\mathrm{Y}$-directions was the same and equal to $2 D$. As seen in Figure 9, the area coverage of the dot arrays is $S / S_{0}=\pi / 16 \approx 0.196$. The calibrating set of dot arrays consisted of eight plates with dot diameters $15 \mu \mathrm{m}, 31 \mu \mathrm{m}, 62 \mu \mathrm{m}, 125 \mu \mathrm{m}, 250 \mu \mathrm{m}, 500 \mu \mathrm{m}, 1000 \mu \mathrm{m}$, and $2000 \mu \mathrm{m}$. The visual appearance of the plates is shown on Figure 10. The microphotographs of the dot arrays are shown in Figure 11. 


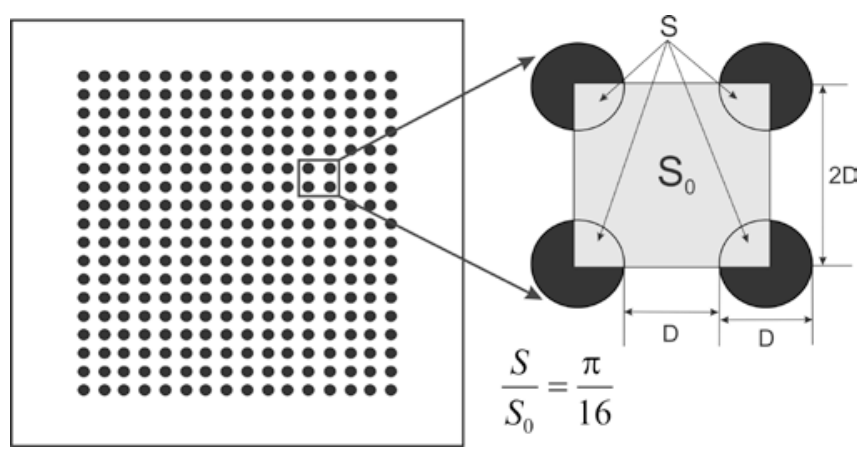

Figure 9. Dot arrays used for calibration of the Cloud Extinction Probe.

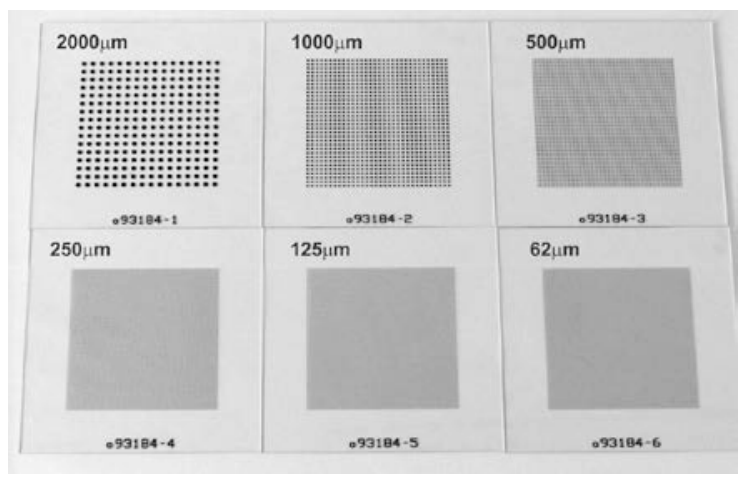

Figure 10. Custom-made calibrating dot grids with different dot diameters. The area coverage of the dot arrays on each grass plate is the same: $S / S_{0}=\pi / 16$.

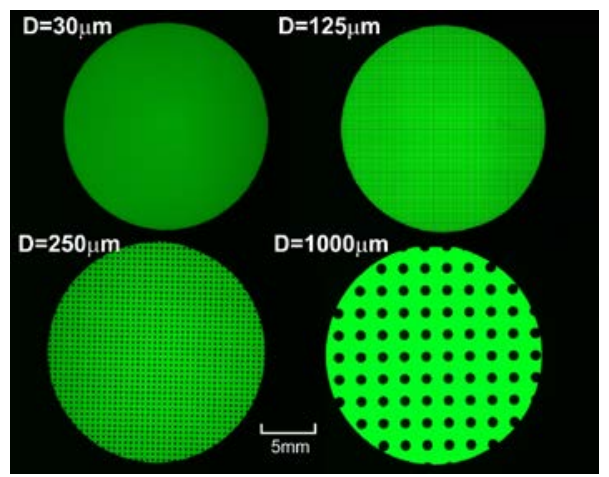

Figure 11. Microphotographs of the dot grids. The diameter of the field of view of the microscope is $20 \mathrm{~mm}$. All dot grids have the same area coverage: $S / S_{0}=\pi / 16$.

The results of calibrations by the dot arrays are shown in Figure 12. It can be seen that extinction efficiency $Q(D)$ approaches 1 for $D>500 \mu \mathrm{m}$, whereas for small dots $Q(D)$ tends toward 2. Such behavior is in good agreement with the theoretical predictions described in Section 6.1. The calibrations of the CEP were also performed with a double-plate scheme as shown in Figure $6 \mathrm{~b}$. The obtained values of $Q(D)$ agreed very well with that obtained for the single-plate scheme (Figure 6a). 


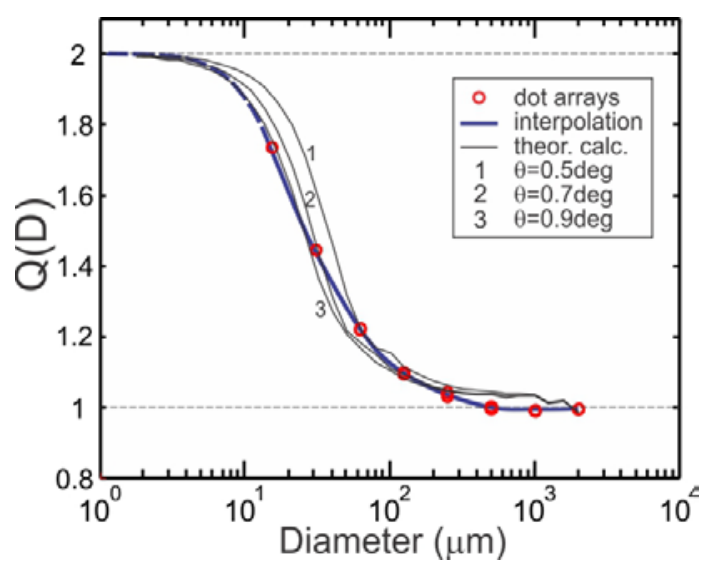

Figure 12. Instrumental extinction efficiency obtained from the CEP calibration by the dot arrays. Black solid curves are theoretical calculations of $Q(D)$ for water spheres for different receiving aperture angles (section 2.1).

The best fit curve $Q(D)$ for the dot arrays lies between the theoretical values calculated for the aperture angles $\theta=0.5 \mathrm{deg}$ and $0.9 \mathrm{deg}$. The deviation from the theoretical predictions may be related to optical misalignment, e.g., the center of the pinhole 4 in Figure 1 is not centered with the optical axis, or other imperfections in the optical elements. Another explanation of this deviation may be related to the fact that the scattering phase functions of the opaque flat discs and those for transparent water spheres are different, and therefore the theoretical calculations in Section 6.1 are not directly applicable to the dot arrays.

The results in Figure 12 justify the approach used for the calibration of transmissiometers with the help of the dot arrays.

\subsubsection{Calibrations by Glass Beads}

Results of the CEP calibrations by opaque flat discs coated on the surface of the glass substrate were presented in the previous section. This approach raises a question about whether these results are applicable to cloud particles, which are essentially three-dimensional and transparent. The forward scattering of such particles may be quite different in comparison to opaque flat particles. Therefore, flat opaque and 3D transparent particles with the same linear sizes may have different instrumental extinction efficiencies $Q(D)$. To address this question, the CEP was calibrated by glass beads. The calibrations were done with monodispersions of glass beads (Thermo Inc.) having nominal diameters: $30 \mu \mathrm{m}, 60 \mu \mathrm{m}$, $70 \mu \mathrm{m}, 120 \mu \mathrm{m}, 230 \mu \mathrm{m}, 480 \mu \mathrm{m}$, and $1000 \mu \mathrm{m}$ with standard deviations $\sigma$ varying between $1 \%$ and $2 \%$.

Calibration by the glass beads consisted of a sequence of the following operations:

1. The calibration started from measurements of the intensity of the beam $\left(I_{\text {glass }}\right)$ transmitted through a glass substrate installed in the center of the CEP beam.

2. Glass beads were positioned at random on the surface of the glass substrate. The surface of the substrate was limited by circular diaphragm with diameter $2 \mathrm{~cm}$. Figure 13 shows pictures of the glass beads with different diameters inside the 2-cm diaphragm.

3. The intensity of beam $I$ attenuated by the glass beads was measured by the CEP. 
4. The microscope was moved in, and a microphotograph of the beads was performed. After the microphotograph was finished, the microscope was moved out of the beam.

5. The beads were blown off the substrate and the background intensity $I_{\text {glass }}$ was measured again.

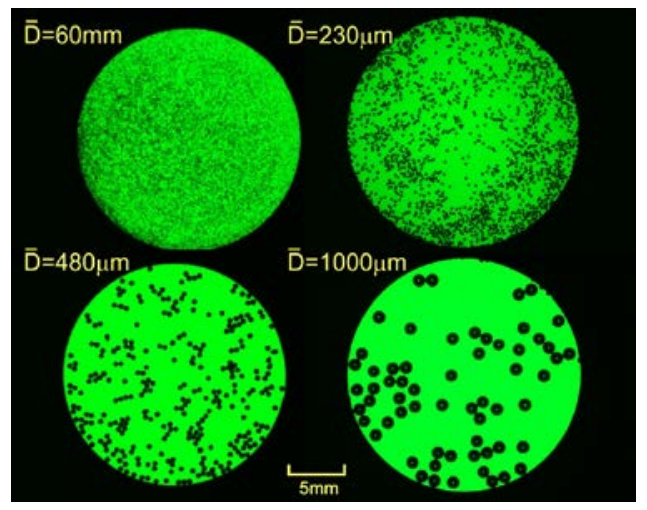

Figure 13. Examples of images of glass beads in the microscope's field of view.

Microscope magnification was selected according to bead size. That way, images of the beads had no less than thirty pixels in diameter. Lower magnification would result in low image resolution and large errors in estimation of the area covered by the images of the beads. For small glass beads, the microscope was set to a high magnification, resulting in a small view field. At high magnification, the microscope view field was reduced, thereby resulting in multiple pictures in order to cover the entire area of the 2-cm diaphragm. Thus, for glass beads with diameter from $30 \mu \mathrm{m}$ to $230 \mu \mathrm{m}$, the selected microscope magnification (1000 pix $/ \mathrm{mm}$ ) required 35 frames in order to cover the entire area. For glass beads with diameter from $200 \mu \mathrm{m}$ to $2 \mathrm{~mm}$, the microscope magnification was set low (230 pix/mm), so that one image frame covered the entire diaphragm area.

Despite the seeming simplicity, the processing of microphotography and calculation of the area coverage $S / S_{0}$ had several significant challenges. First, microscope images are prone to different optical aberrations, which result in the deformation of images of beads, thus influencing estimation of their areas. The most significant of these aberrations contributing to the errors in $S / S_{0}$ is the distortion aberration. Figure 14c explains the effect of the distortion aberration, which usually increases with decreasing microscope magnification; at high magnification, the effect of distortion becomes insignificant. The effect of the distortion aberration also varies over the microscope's field-of-view, increasing towards the periphery.

Second, image size is sensitive to the threshold intensity level $I_{\text {thresh }}$ selected for the particle image sizing (Figure 14b). Thus, the size of the image increases with increasing $I_{\text {thresh }}$.

Third, background illumination intensity varies over the microscope's field-of-view, with intensity decreasing towards the outer edges. The non-uniform background intensity affects a change in particle image size depending on its position in the microscope's field-of-view. As such, if constant $I_{\text {thresh }}$ is used for the entire image frame (Figure 14a), then after conversion of the grayscale image into black-andwhite, the size of monodisperse particles in the center will be smaller than those in the periphery (Figure 14c). 
The last two issues turned out to have the largest contribution to the accuracy of calculation of $S / S_{0}$ in the experimental setup. Figure 15 shows the effect of the three above issues on the calculation of particle size distribution for the case of a $125 \mu \mathrm{m}$ dot array.

In order to overcome these problems, the following procedures were applied during image processing. The effect of the distortion aberration was accounted for with the help of the retrieval matrices $C$ obtained from the microphotographs of the images of the dot arrays. The corrections were applied with the help of Matlab function imtransform(Im,C) from the Image Processing Toolbox. This function applies spatial transformation of the image Im with the rule established in accordance with the correction matrix $C$.

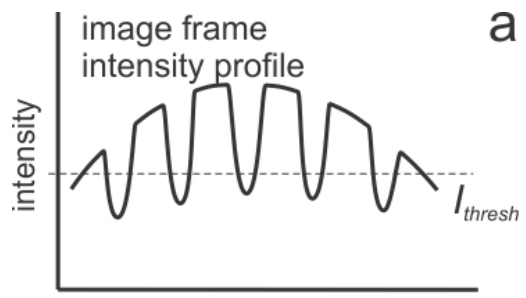

distance
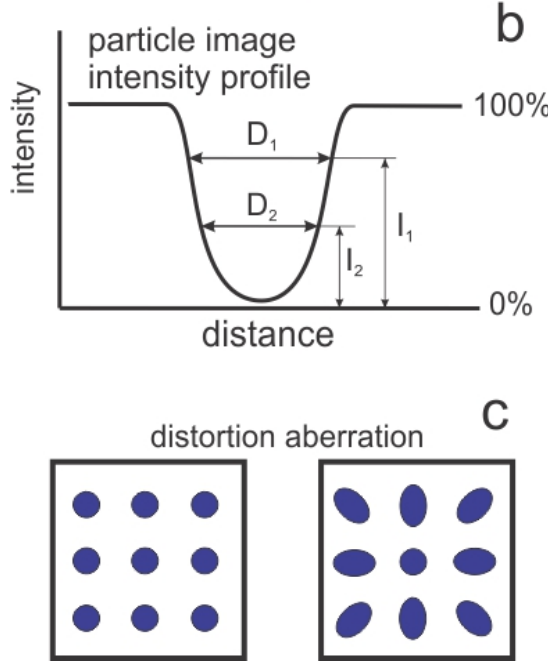

Figure 14. Explanation of different effects causing errors in sizing of images of particles: (a) inhomogeneity of background intensity, (b) different threshold intensity in conversion of grayscale into b/w image, (c) aberration of distortion.
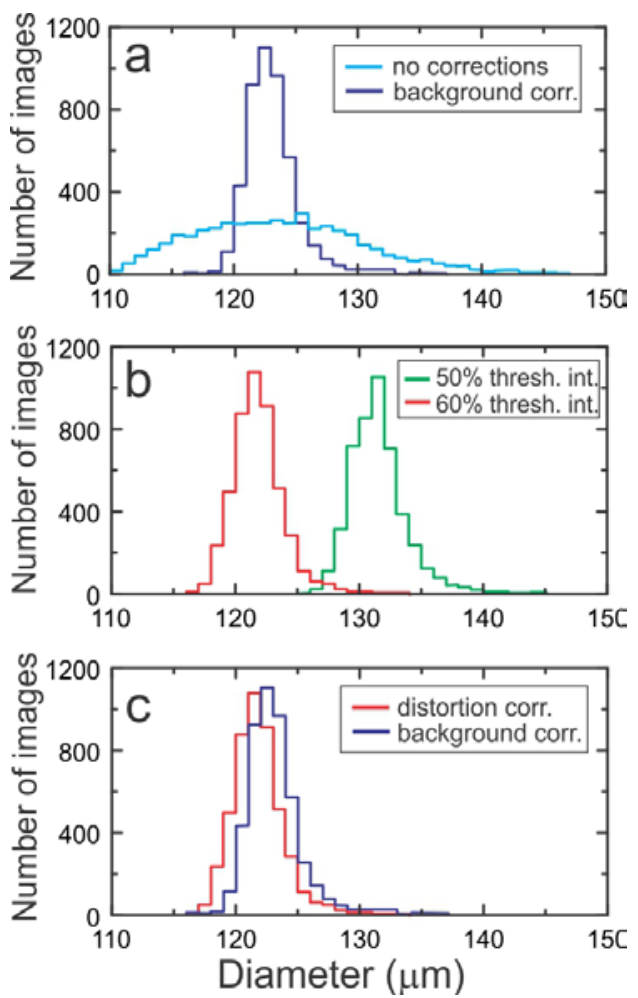

Figure 15. Contribution of different corrections on the reconstructed size distribution deduced from the analysis of the image frame of the $125 \mu \mathrm{m}$ dot grid. (a) Corrections on the inhomogeneity of background intensity, (b) effect of the threshold intensity on image sizes, (c) effect of the distortion aberration corrections.

The effect of the background illumination was accounted for by either (1) normalizing the background intensity with the help of an image without beads or (2) splitting the original image frame into $12 \times 16$ sub-frames and assuming that the background intensity within each sub-frame is constant. The first approach is very time-consuming and requires significant computer resources, and it was applied only for a single frame of measurements. For 35-frame cases, the processing time of one set of microphotographs 
on a computer exceeds one day. The second approach was used for the cases with multi-frame measurements.

The cut-off level for image sizing was determined as a middle point between the maxima corresponding to the background and particle shadows on the intensity distributions of all pixels in the grayscale image frame (or sub-frame). Figure 16 provides an explanation of the determination of the cut-off level of the image frames. Determination of the cut-off level this way corresponds to particle sizing at approximately $50 \%$ threshold intensity level.

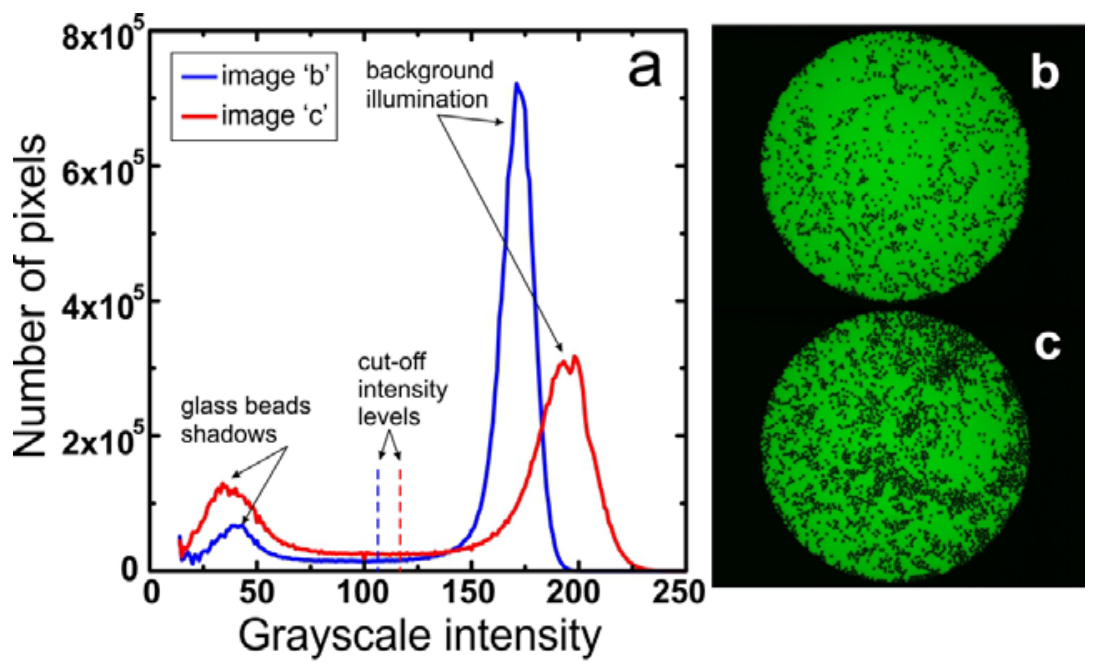

Figure 16. Determination of the cut-off level for conversion of a grayscale image into black-and-white for subsequent particle sizing and determination of particle area coverage.

The final tuning of the corrections described above and identifying cut-off levels was accomplished with the help of the microphotographs of the dot arrays. After applying the corrections, the accuracy in determining $S / S_{0}$ is estimated to be $5 \%$ to $10 \%$ depending on bead size and microscope magnification. If the corrections are not applied, the systematic errors in calculations of $S / S_{0}$ may reach $30 \%$.

The sequence of operations in processing and analysis of multi-frame microphotography of the substrate with the glass beads is shown in Figure17. Finding common patterns in the neighboring image frames and subsequent frame concatenation was performed with a special program written in Matlab. 
original set of successive

microphotographs of the target

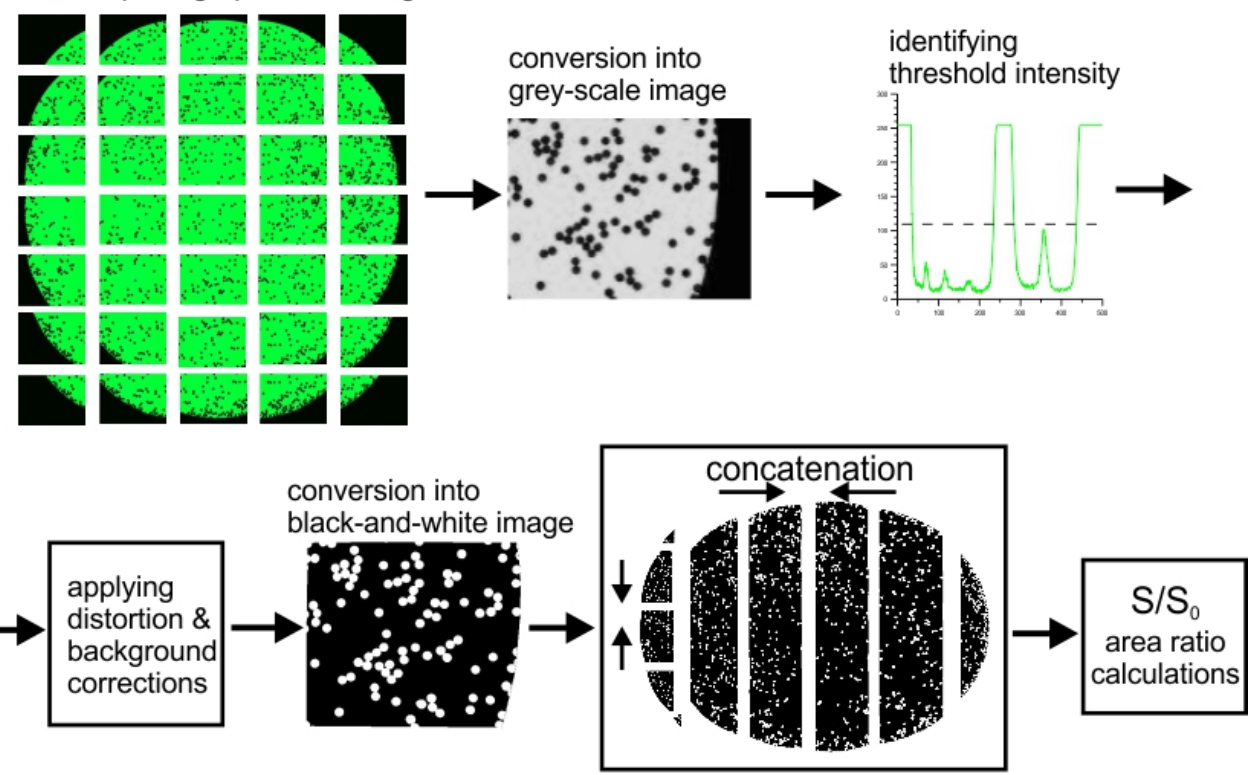

Figure 17. Sequence of procedures during image processing and calculation of $S / S_{0}$.

Figure 18 shows the results of the CEP calibrations for glass beads. The total number of calibration points is 75 for different sizes of glass beads. As seen from Figure 18, instrumental extinction efficiencies obtained for the glass beads are in good agreement with $Q$ obtained for the dot arrays, which justifies the use of the constant frequency grids for the calibration of transmissiometers.

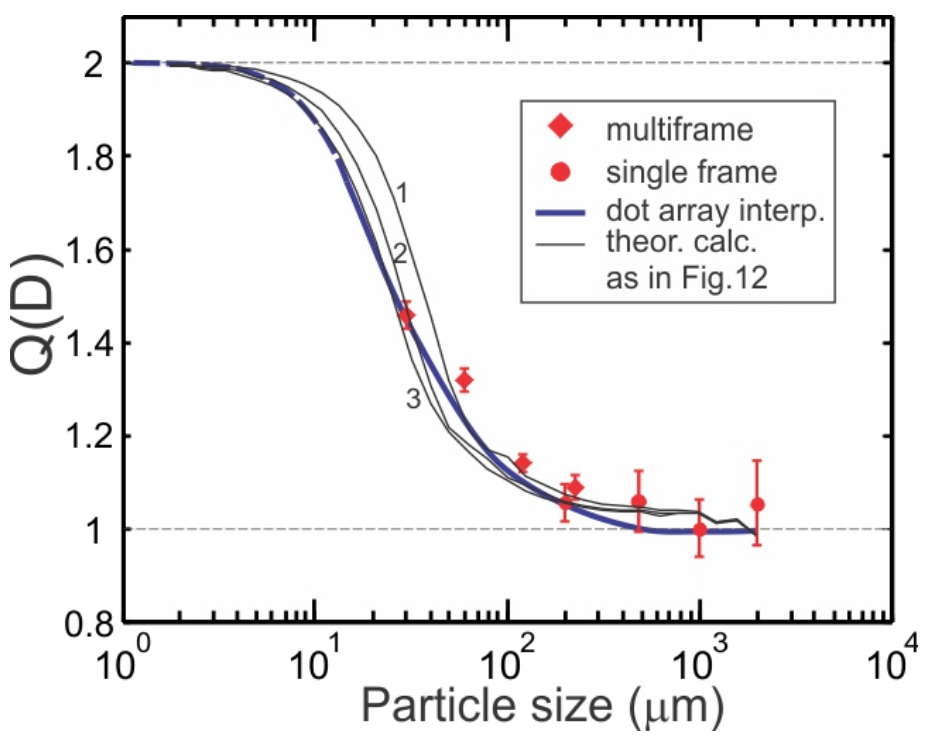

Figure 18. Instrumental extinction efficiency versus diameter calculated from the CEP calibrations for monodisperse glass beads. 


\subsubsection{Calibrations by Constant Frequency Stellar Grids}

The results obtained in the previous two sections show the importance of the dispersion of the attenuating particles for the measured extinction coefficient. For example, for two sprays composed of particles with different sizes, but having the same total projected area, the measured extinction coefficient for the spray with the larger particles may be lower than that of the smaller ones.

This raises a question about the role of characteristic sizes of elements composing a particle on the measured light attenuation. For example, if a 1-mm-size ice particle consists of an assembly of $100 \mu \mathrm{m}$ ice crystals, then will it attenuate light as a $100-\mu \mathrm{m}$ or a 1-mm particle? What are the elements composing a particle that determine its forward scattering? These questions are important for understanding what the characteristic size of non-spherical particle should be used upon applying corrections to measured extinction efficiencies.

To address this question, a set of custom-made constant frequency arrays consisting of six-ended stars were used. The six-ended stellar shape was chosen because it is one of the common habits of ice crystals. Since the characteristic sizes of the fine structure of stellar branches are usually much smaller than the size of the particle, it is not clear what the attenuated properties of such particles would be. For this particular case, the thickness of the stellar branches was $0.1 L$, where $L$ is the length of the branch. Figure 19 shows the pattern of the custom-made stellar arrays. Distance between the centers of the stars was $1.25 L$, so the area ratio is $S / S_{0}=0.1772$. Three stellar arrays with sizes $L=500 \mu \mathrm{m}, 1000 \mu \mathrm{m}$, and $2000 \mu \mathrm{m}$ were used. Microphotographs of the stellar arrays are shown in Figure 20.

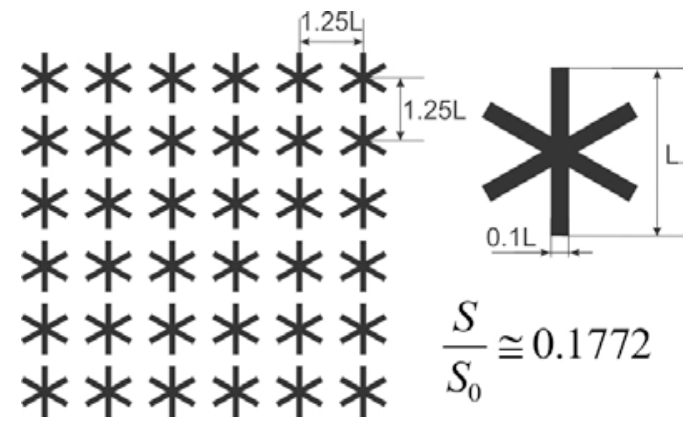

Figure 19. Arrangement of the stellar patterns on the "stellar" grids.

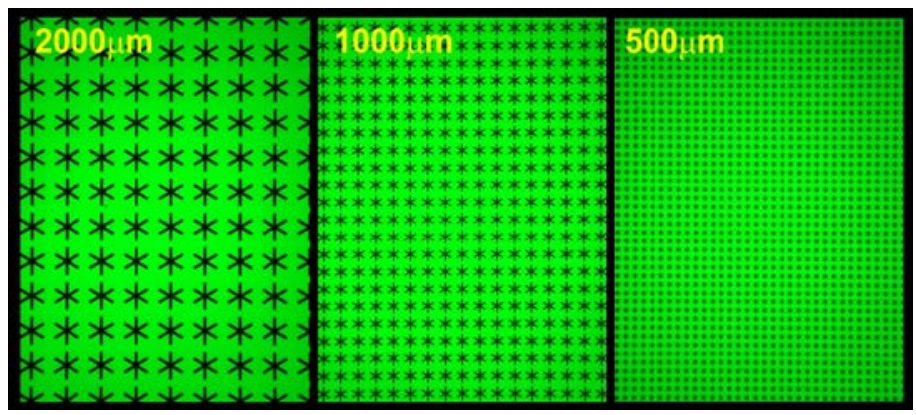

Figure 20. Visual appearance of the stellar grids under microscope.

Figure 21 shows the instrumental extinction efficiency for the stellar habits. Since the considered objects have non-circular shapes, there may be several different definitions of their size. On the diagram in 
Figure 21, the values of $Q$ were plotted versus four characteristic sizes: (1) maximum stellar dimension $D_{\max }=L$, (2) effective size $D_{\text {eff }}=\sqrt{4 S / \pi}$, (3) minimal size $D_{\min }=0.1 L$, which represents the width of the stellar branch, and (4) best-fit size $D_{\text {fit }}=2 D_{\min }=0.2 L$. As seen from Figure 21, utilizing $D_{\max }$ and $D_{\text {eff }}$ results in overestimation of $Q$, whereas $D_{\min }$ would underestimate $Q$. However, $Q(D)$ obtained for dots would agree with that for stellar shapes for $D_{\text {fit }}=0.2 L$. In other words, in terms of the attenuation measured by the CEP, a stellar particle with size $L=500 \mu \mathrm{m}$ behaves like an opaque disc with $D=100 \mu \mathrm{m}$.

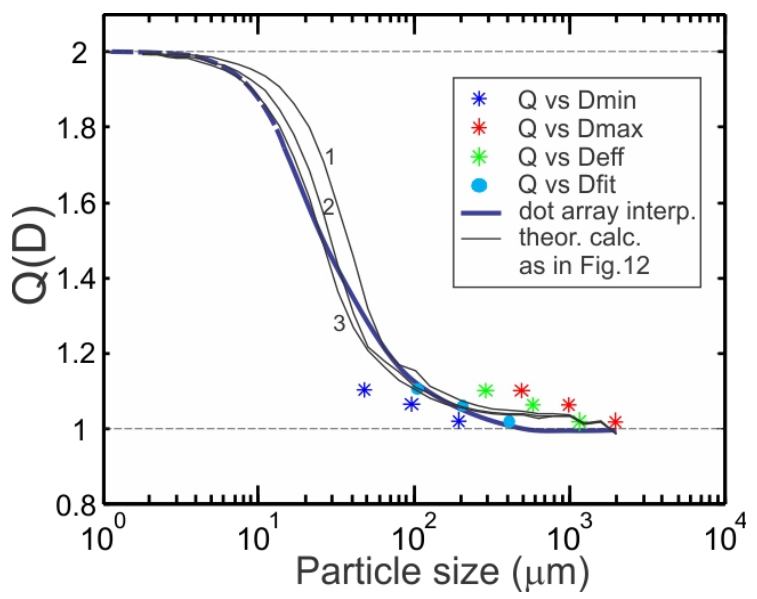

Figure 21. Instrumental scattering efficiency obtained from the CEP calibrations by stellar arrays with three different sizes: $L=500 \mu \mathrm{m}, 1000 \mu \mathrm{m}$, and $2000 \mu \mathrm{m}$. Due to the ambiguity of the size definition of non-circular particles, different sizes were applied to the stellar habits.

\subsubsection{Calibrations by Irregular-shaped Particles}

Calibrations by the stellar arrays in Section 6.2.6 suggest that the function $Q(D)$ for non-spherical particles (non-circular projected area) may be quite different from $Q(D)$ obtained for spherical particles. The reasons for these differences are related to the ambiguity of the definition of particle size for nonspherical particles and the difference in scattering functions of non-spherical and spherical particles. The following set of experiments attempts to identify feasibility of finding the size of non-spherical particles that would be universal for different habits for use in $Q(D)$ and thus used for corrections to extinction coefficient.

Crystals of sugar and broken glass particles were used as optical targets in order to simulate attenuation by cloud ice particles. Figure 22 shows two microphotographs of sugar crystals scattered over the glass substrate (left) and their corresponding distributions of $X, Y$, and $D_{\text {eff }}$ (right). Here, $X$ and $Y$ are randomly oriented Cartesian coordinates in the image plane during image processing. As seen from the diagrams in Figure 24, all three distributions and their modal sizes are in a good agreement with each other. The modal sizes for all three size definitions range from $600 \mu \mathrm{m}$ to $800 \mu \mathrm{m}$. Figure 22 shows that all points $Q(D)$ are well grouped and are in good agreement with the results for dot arrays. 

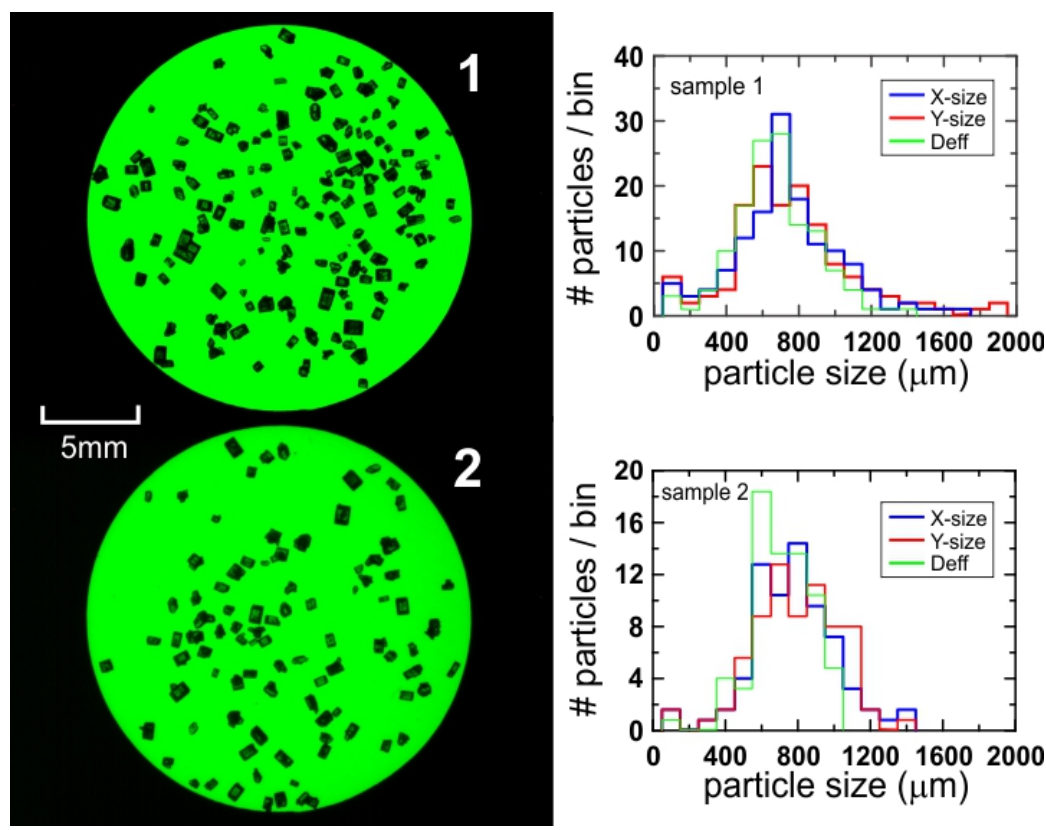

Figure 22. Microphotographs of sugar crystals (left) and corresponding distributions of $X, Y$, and $D_{\text {eff }}$ sizes (right).

Figure 23 shows three microphotographs of broken glass particles (left) and corresponding distributions of $X, Y$, and $D_{\text {eff }}$ sizes (right). The particles in cases 2 and 3 have transparent sections. Such transparent areas are typical for many types of ice crystals. Due to the transparent sections, the effective size $D_{\text {eff }}$ of the broken glass particles is smaller than $D_{x}$ and $D_{y}$. However, for case 1 in Figure 23, all three size distributions agree, because the particle images appear to be mostly without holes and transparent areas.

Figure 24 shows that extinction efficiency for broken glass particles turned out to increase with increasing particle size. Such behaviour of $Q(D)$ appears to be opposite to that for glass beads and dot arrays. For example, the scattering efficiency of $2-3 \mathrm{~mm}$ glass particles would be the same as that for approximately $30 \mu \mathrm{m}$ glass beads or dots. It should be noted that the transparent sections of the images of the glass particles were not included in the calculations of $S / S_{0}$. A potential explanation of this may be related to the transparent sections in the broken glass imagery, since this is the only distinct difference with the previous targets. This may result in a flatter forward-scattering lobe and increase of the instrumental scattering efficiency. 

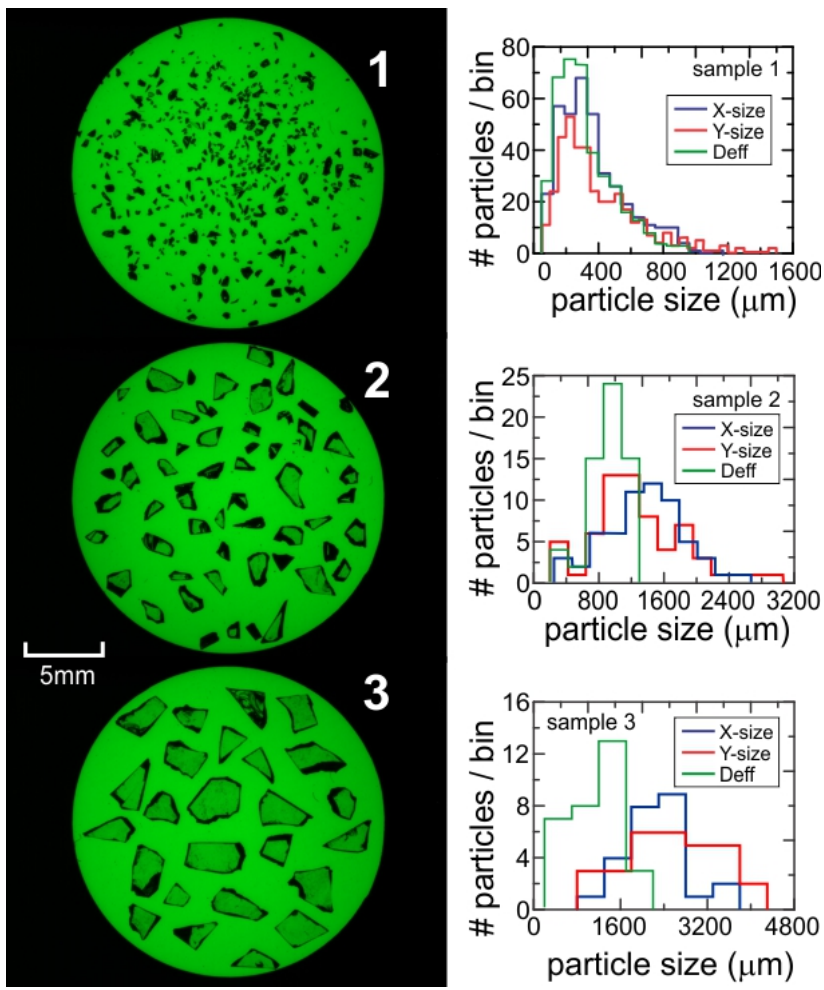

Figure 23. Microphotographs of broke glass particles (left) and corresponding distributions of $X, Y$, and $D_{\text {eff }}$ sizes (right).

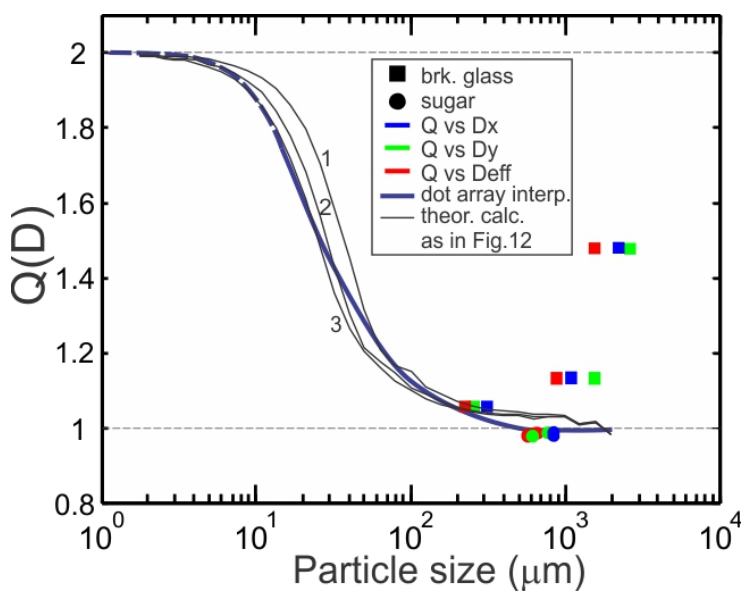

Figure 24. Instrumental scattering efficiency obtained from the CEP calibrations for sugar crystals and broken glass particles as shown in Figures 22 and 23. 


\subsection{Laboratory Studies of the CEP Performance}

\subsection{Effect of the Beam Inhomogeneity}

Attenuation in transmissiometers may depend on particle position along the beam direction. In order to estimate this effect, light attenuation was measured versus the distance of the dot arrays from the CEP front lens. It was found that the effect of the CEP response versus the target position increases as particle size decreases. Thus, for the $15-\mu \mathrm{m}$ dot array, the CEP response deviates $+/-2.5 \%$ from its average value, whereas for the $2000-\mu \mathrm{m}$ dot array, it is only $+/-0.2 \%$ (Figure 25). Therefore, in general, the dependence of attenuation versus position along the beam can be considered as negligible, and it is not expected to cause significant biases in measured extinction coefficient.
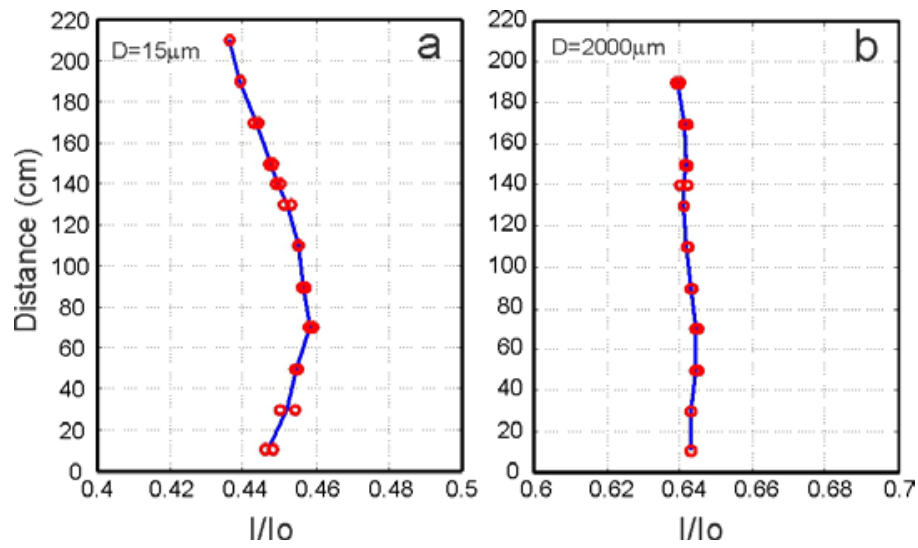

Figure 25. CEP output signal versus position along the beam direction of the $15-\mu \mathrm{m}$ (a) and $2000-\mu \mathrm{m}$ (b) dot arrays.

Figure 26 shows changes of the CEP signal versus positioning of the retroreflector across the beam. It shows that the signal changes approximately $3 \%$ when the retroreflector is displaced $7 \mathrm{~mm}$ from the center of the beam. Figure 26 allows estimation of the effect of vibration and mutual displacement of the retroreflector and optical unit on the noise of the output signal. The ground tests showed that the displacement of the optical unit and retroreflector does not exceed $+/-2 \mathrm{~mm}$. It turned out that the observed inhomogeneity of the beam does not produce any significant effect of the signal noise. During flight operation, it was found that the noise of the extinction coefficient caused by airframe vibrations does not exceed approximately $0.1-0.2 \mathrm{~km}^{-1}$.

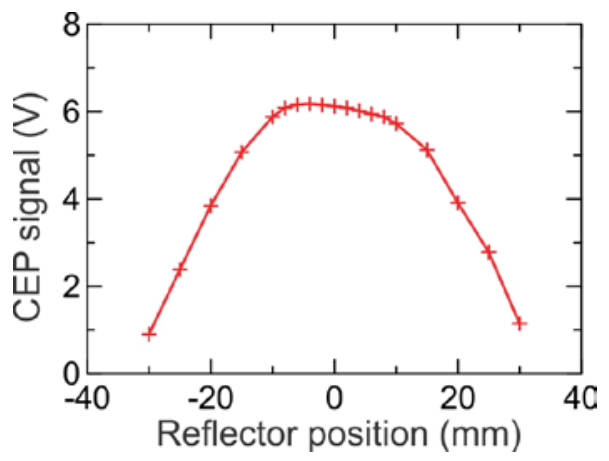

Figure 26. CEP output signal versus position of the retroreflector across the beam. 


\subsection{Temperature Effect on the CEP Performance}

The environment inside the CEP optical unit is temperature-controlled, and the heaters have sufficient power to maintain a constant temperature of $+20^{\circ} \mathrm{C}$ inside the CEP down to $-30^{\circ} \mathrm{C}$ outside temperature. Below $-30^{\circ} \mathrm{C}$, the CEP inside temperature starts decreasing. There are few elements in which sensitivity to temperature may be critical to the performance of the probe. The most important of these elements are the LED and bandpass filter. The effect of temperature changes on performance of the CEP was tested in the cold room. The experimental setup of the cold room tests is shown in Figures 27 and 28.

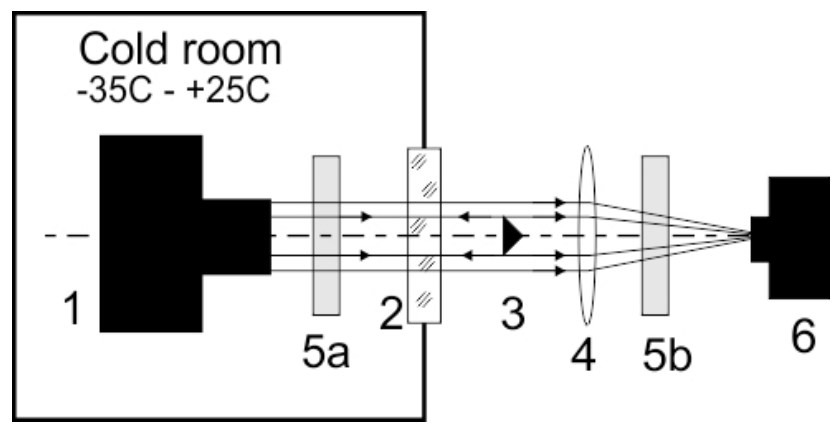

Figure 27. Experimental setup for the temperature sensitivity tests in the cold room: (1) CEP, (2) optical window, (3) retroreflector, (4) condenser lens, (5ab) bandpass filter, and (6) optical spectrophotometer.

During the experiment the CEP heaters were disconnected, and the temperatures of the LED and the bandpass filter equaled the air temperature inside the cold room. The bandpass filter used in the experiment had the same specifications as those installed in the optical scheme in front of the photodetector (Figure 1). Depending on the experiment, the bandpass filter was installed either inside the cold room (5a) or outside (5b) (Figure 27).

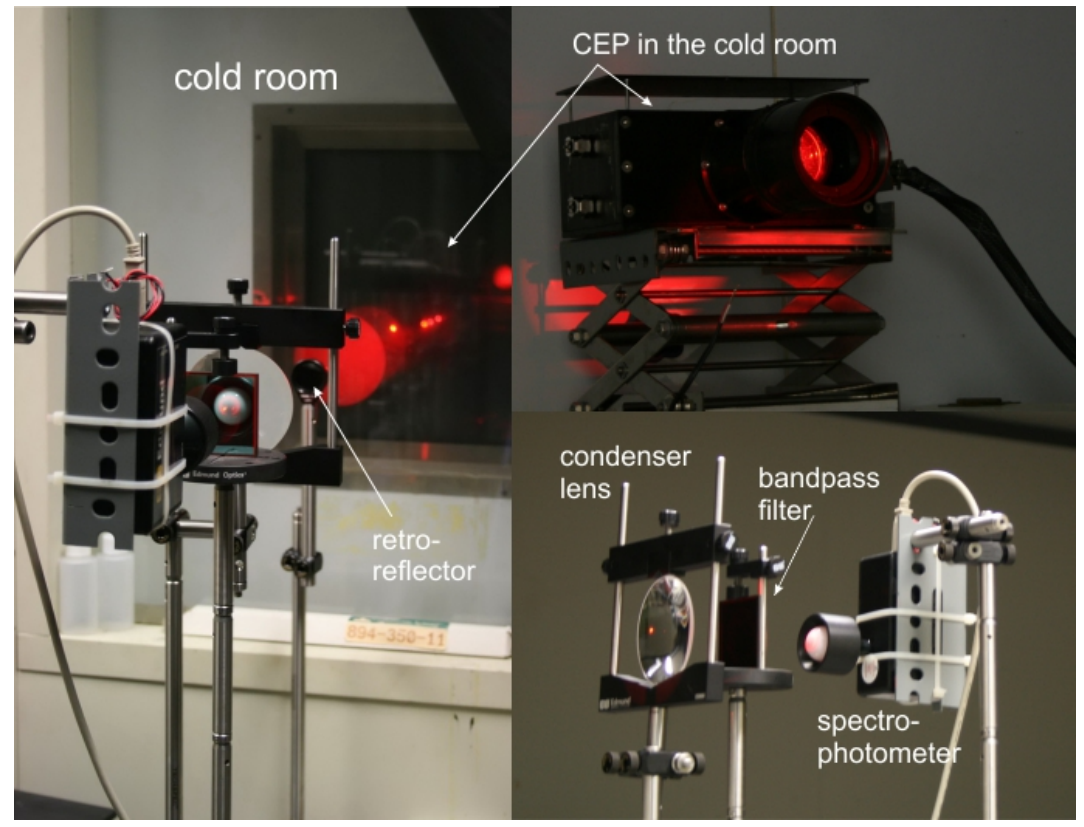

Figure 28. Pictures of the elements of the experimental setup shown in Figure 27. 
Figure 29 shows the spectral bandpass filter transmittance at two temperatures $\mathrm{T}=-30^{\circ} \mathrm{C}$ and $+15^{\circ} \mathrm{C}$. In this type of experiment, the bandpass filter was mounted inside the cold room, and an incandescent lamp was used as a source of radiation. As seen in Figure 29, the changes of the spectral transmittance of the bandpass filter are quite small.

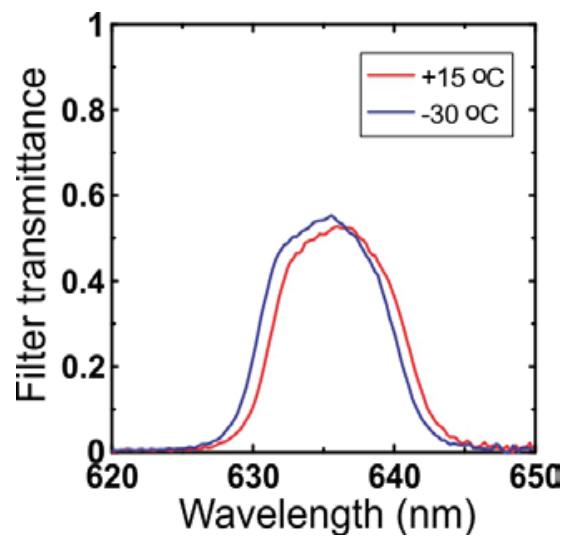

Figure 29. Spectral transmittance of the bandpass filter installed in the CEP.

The temperature dependence of the LED was studied for the range $-24^{\circ} \mathrm{C}<\mathrm{T}<+25^{\circ} \mathrm{C}$. Figure 30 shows the dependence of the LED emitted spectrum versus temperature. In accordance with the general behavior the LED, emissivity increases with decreasing temperature. However, it was found that the measured spectral irradiance as shown in Figure 30 is quite different from that declared by the manufacturer. The spectral irradiance of the light passed through the bandpass filter does not experience any significant changes.

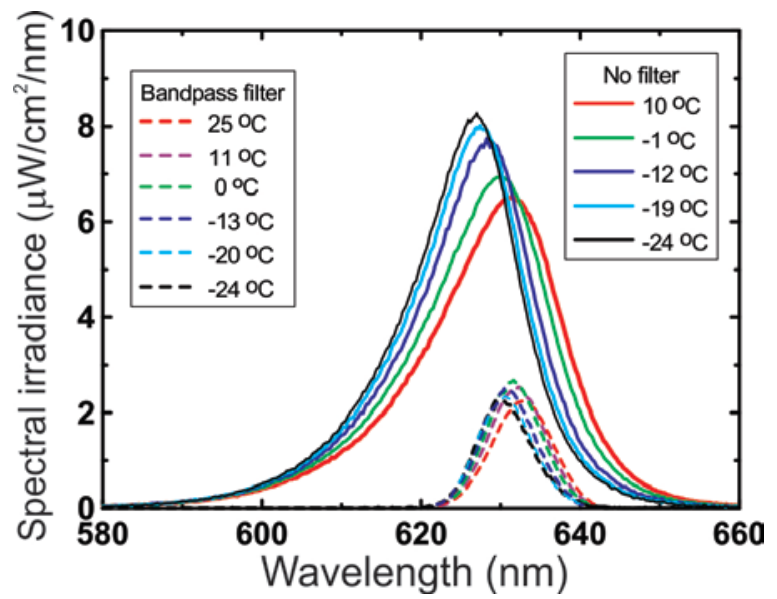

Figure 30. Spectral irradiance of the LED measured at different temperatures.

Figure 31 shows LED spectral irradiance, $\lambda_{\max }$ and spectral width deduced from the spectral irradiances shown in Figure 30 The spectral width changes less than $2 \mathrm{~nm}$ (Figure 31a), and the wavelength corresponding to maximum irradiance $\lambda_{\max }$ changes approximately $3 \mathrm{~nm}$ (Figure 31b). As seen from Figure 31c, the LED irradiance stays nearly constant in the considered temperature interval. Taking into account that the attenuation of the light, LED emissivity, and background radiation are measured with the same photodetector, such small temperature dependence of the above parameters cannot, in any significant way, affect the performance of the CEP. 

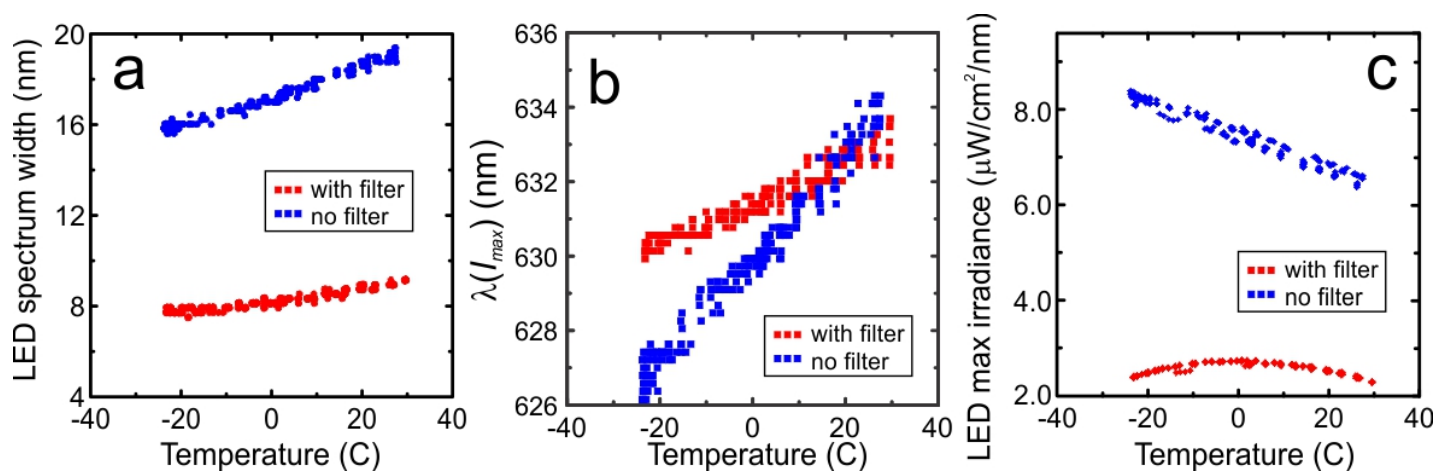

Figure 31. LED spectrum width (a), wavelength corresponding to the max irradiance in the LED spectrum (b), LED max irradiance (c) deduced from the data shown in Figure 30.

\subsection{Polarization}

Ice particles may significantly polarize transmitted light. Therefore, if a transmissiometer receiver has non-isotropic polarization sensitivity, the result might be a reduction of the measured intensity of transmitted light and thus an underestimation of extinction coefficient. One potential source of polarization of the emitted and received light is a beam-splitter (7) in Figure 1. The polarization sensitivity of the CEP was tested with the help of the polarizer mounted between the CEP optical unit and the retroreflector. Figure 32 shows that the difference in measurements of polarized light does not exceed $3 \%$. This is quite a small value that does not require any special consideration in data analysis.
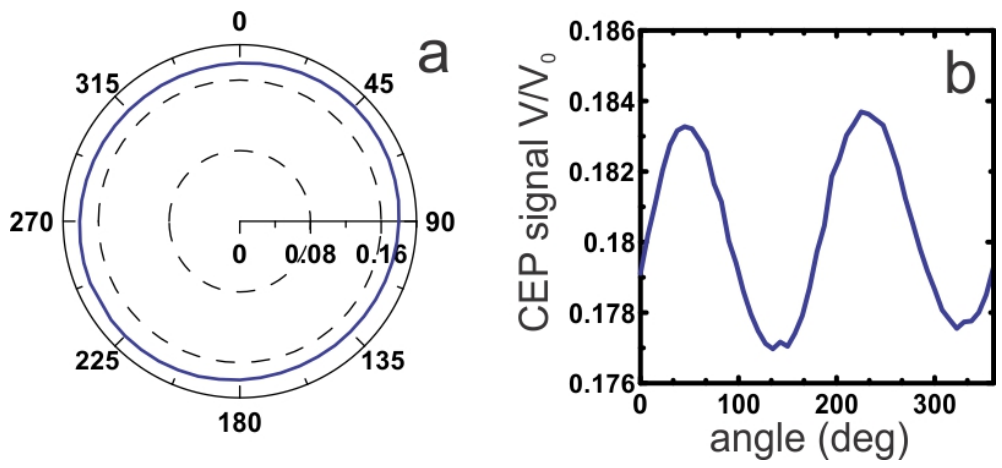

Figure 32. Polarization sensitivity of the CEP in (a) polar and (b) Cartesian coordinates.

\subsection{CEP Measurements during ISDAC}

The extinction coefficient measured by the CEP was calculated based on the Beer-Bouguer law as

$$
\beta_{C E P}=-\frac{1}{2 L} \ln \frac{I}{I_{0}}
$$

Here $I$ and $I_{0}$ are the output signals that characterize the radiant fluxes transmitted in clouds and clear sky, respectively. The intensity of the attenuated signal was calculated as $I=I_{t o t}-I_{b k g}-I_{\text {int }} ; I_{0}$ was determined the same as $I$ but in a cloud-free atmosphere. The signals $I_{t o t}, I_{b k g}$ and $I_{\text {int }}$ were normalized on the current values of $I_{\text {norm }}$. 


\subsection{Calculations of the Extinction Coefficient from FSSP and CDP Measurements}

In liquid clouds, the extinction coefficient was calculated from the FSSP droplet-size distribution measured in fifteen size bins as

$$
\beta_{F S S P}=\frac{\pi Q}{4} \sum_{j=1}^{15} n_{j} D_{j}^{2}
$$

where $n_{i}, D_{i}$ are concentration and diameter of droplets in the FSSP $i$-th size bin, and $Q$ is extinction efficiency. Since the size of the FSSP measured droplets $D>>\lambda, Q \approx 2$ is a good approximation.

\subsection{Calculations of the Extinction Coefficient from Imaging Probes}

Extinction coefficient for ice clouds was calculated from Optical Array Probe (OAP) imagery. OAPs provide shadowgraphs of cloud particles that passed though the sample area of the probe (Figure 33a). In general, the OAP can be considered to be an extinctiometer, but instead of measuring attenuation of light integrated over the whole beam, it measures local attenuation associated with the discrete binary images with shadow areas $A_{j}$ (Figure 33b). Therefore, extinction coefficient can be calculated through the integration of the area shadowed by all particles $\sum A_{j}$ as,

$$
\beta_{O A P}=\frac{Q}{L A_{0}} \sum_{j} A_{j}
$$

Here $L$ is the distance between the OAP arms (Figure 33b); $A_{0}$ is the total area covered by the probe's laser beam having width $W$ and moving at speed $U$ during time $\Delta t$, i.e., $A_{0}=W U \Delta t$. Substituting this expression into Eq.29 yields

$$
\beta_{\text {OAP }}=\frac{Q}{L W U \Delta t} \sum_{j} A_{j}
$$

The direct area calculation (DAC) technique for estimating extinction coefficient is based on the following assumptions regarding OAP imagery: (1) the depth-of-field and the sample area width do not depend on particle size, i.e., the sample area of the probe stays constant for all particles; and (2) the shadow images represent geometrical shadows of cloud particles and so diffraction effects are neglected. 

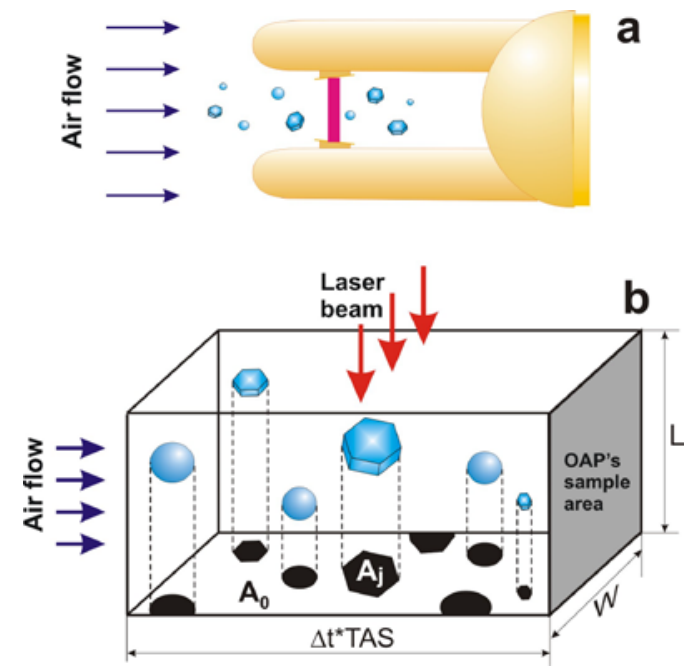

Figure 33. Conceptual diagram of calculation of extinction coefficient from OAP-2D imagery.

Assumption (1) is satisfied for particles with $D \geq 125 \mu \mathrm{m}$ for OAP-2DC and for particles with $D \geq 400 \mu \mathrm{m}$ for OAP-2DP, i.e., when the depth-of-field for these particles is larger than the distance between the arms. Korolev et al. (1998) showed that the projected image area experiences several oscillations when a particle moves from the object plane to the edge of the depth-of-field. The effect of particle distance on projected area decreases with increasing particle size. In other words, the DAC technique is expected to work better for larger particles than for small ones. It should be mentioned that calculation of extinction coefficient from OAP-2DP/2DP imagery for ice clouds with large concentrations of ice particles $(D<100 \mu \mathrm{m})$ may result in significant underestimation of the extinction coefficient.

The DAC method gives a more accurate estimation of the extinction coefficient, as compared to the alternative method based on the size-to-area conversion (STAC)

$$
A=a D^{b} .
$$

Sources inaccuracy for the STAC method are related to uncertainty in the coefficients $a$ and $b$ for different particle habits. The STAC method also cannot be applied to partial images, and this significantly limits its use for particles with $D>W$.

\subsection{Comparisons of the CEP and Particle Probe Data in Liquid Clouds}

Figure 34 shows time series of the extinction coefficient for a low-level stratiform deck sampled on Flight 30 of the ISDAC field campaign. The high frequency of cycling of the Rosemount Ice Detector (RICE) signal (Figure 34b) indicates that the cloud contained supercooled liquid water. Figure 34a shows that extinction coefficient measured by the CEP and that calculated from the FSSP and CDP varied from approximately 0 to $60 \mathrm{~km}^{-1}$. While this cloud layer also contained some ice particles, estimations from the OAP-2DC/2DP imagery suggest that for most of the cloud, extinction coefficient associated with ice is less than $0.5 \mathrm{~km}^{-1}$, much smaller than that for liquid regions. Therefore, this cloud layer can be considered as conditionally liquid, and the effect of ice particles on extinction coefficient measured by the CEP, FSSP, and CDP can be neglected. 


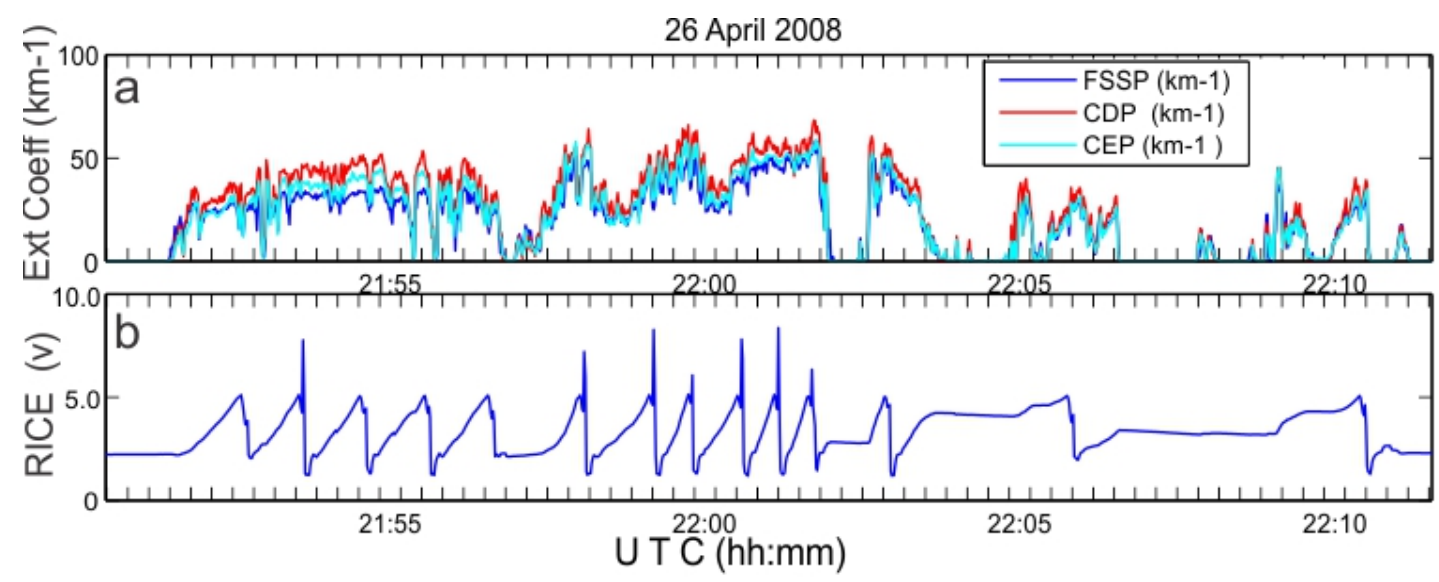

Figure 34. Time series of the measurements of extinction coefficient by the CEP and particle probes FSSP and CDP (a), and Rosemount Icing Detector signal (b). Measurements were made in a stratiform cloud deck during the ISDAC field campaign in the Cape Barrow region, at $900<\mathrm{P}<980 \mathrm{mb} ;-11<\mathrm{T}<-8 \mathrm{C}$, Flight 30, 26 April 2008.

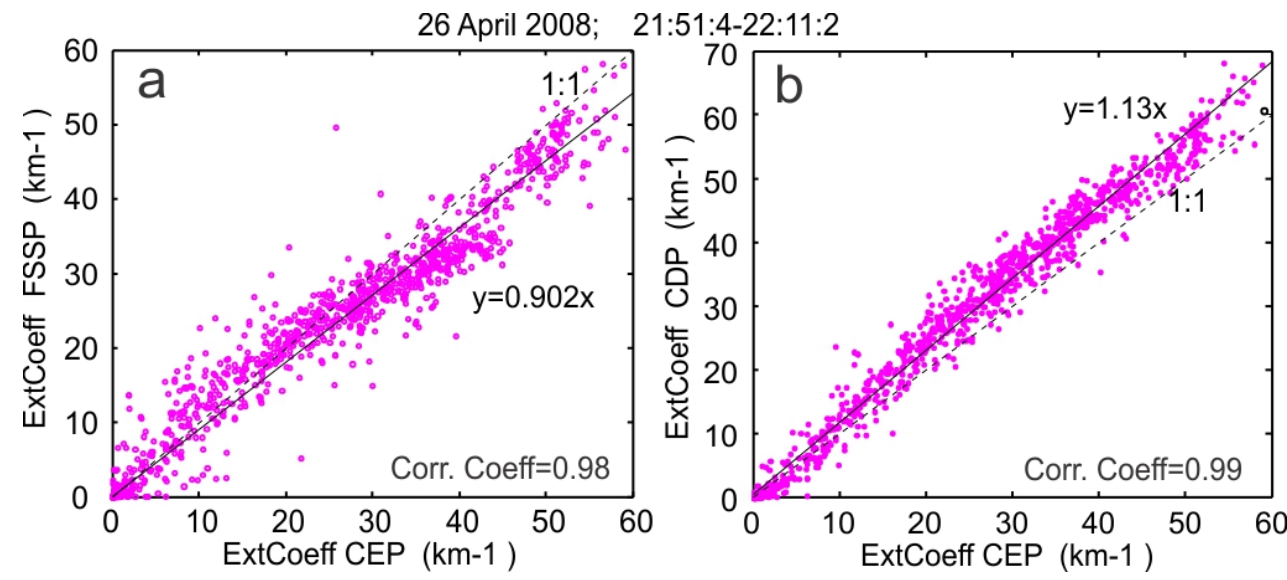

Figure 35. Scatter diagrams of extinction coefficient measured by CEP and particle probes FSSP (a) and CDP (b) in liquid clouds on 26 April 2008 during the ISDAC field campaign (Figure 34).

Figure 35 shows scatter-diagrams between CEP, FSSP, and CDP measurements of extinction coefficient. For liquid clouds, the extinction coefficients measured by the CEP and particle probes FSSP and CDP agree reasonably well with each other. The scattering of the points does not exceed $15 \%$. Such agreement is anticipated since as seen from Figure 36a, the main contribution to extinction coefficient is made by droplets with $D<15 \mu \mathrm{m}$. The CEP instrumental extinction efficiency for such droplets is close to 2 (Figure 36b). Thus, results from in situ measurements in liquid clouds provide good closure with laboratory experiments.

The results of comparisons between airborne transmissometers and particle probes in liquid clouds similar to those shown in Figures 34 and 35 were also discussed in Korolev et al. (1999) and Korolev (2008). 

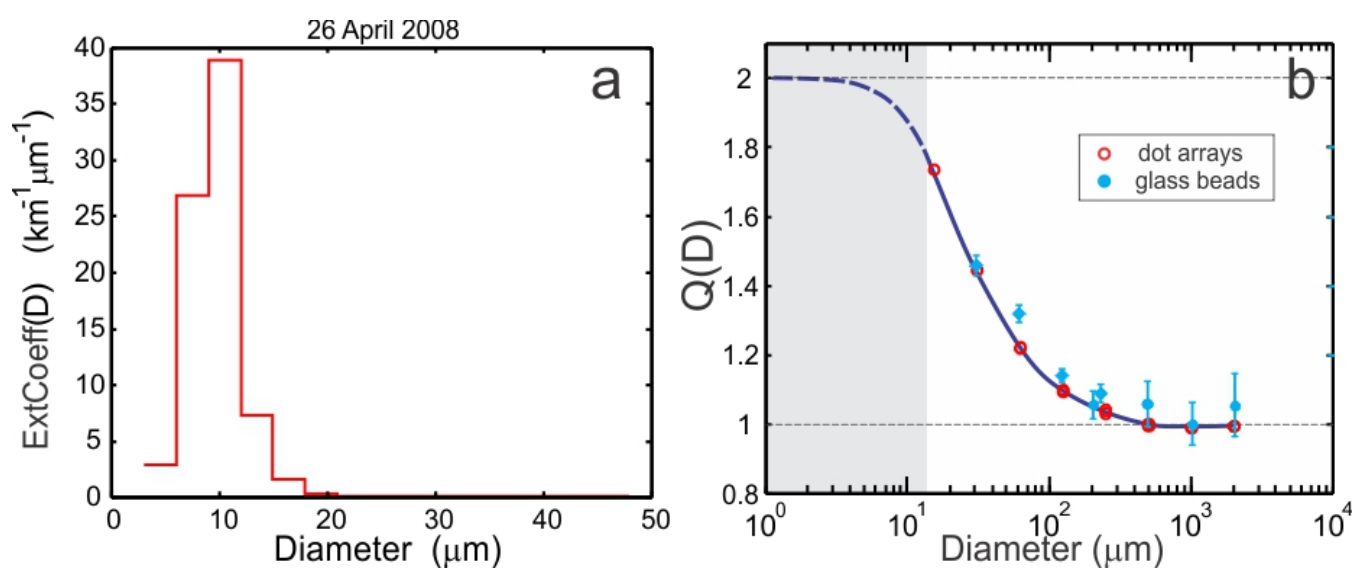

Figure 36. Distribution of extinction coefficient measured by FSSP and averaged over the time period corresponding to that shown in Figure 34 (a). Gray area indicates region of the instrumental extinction efficiency corresponding to the droplets mainly contributing to extinction coefficient based on (a).

\subsection{Comparisons of the CEP And Particle Probe Data in Ice}

Figure 37 shows spatial variations of extinction coefficient measured by CEP, OAP-2DC, and OAP-2DP during a flight through As-Cs clouds. Extinction coefficients deduced from the 2D probes were calculated using DAC technique (Eq.30). Small variations of the RICE signal indicate the absence of liquid along the flight line (Mazin et al. 2001). This helps identify this cloud as glaciated. Images measured by the OAP-2DC shown in Figure 38b suggest that most ice particles were bullet rosettes with maximum sizes between $1 \mathrm{~mm}$ and $2 \mathrm{~mm}$.

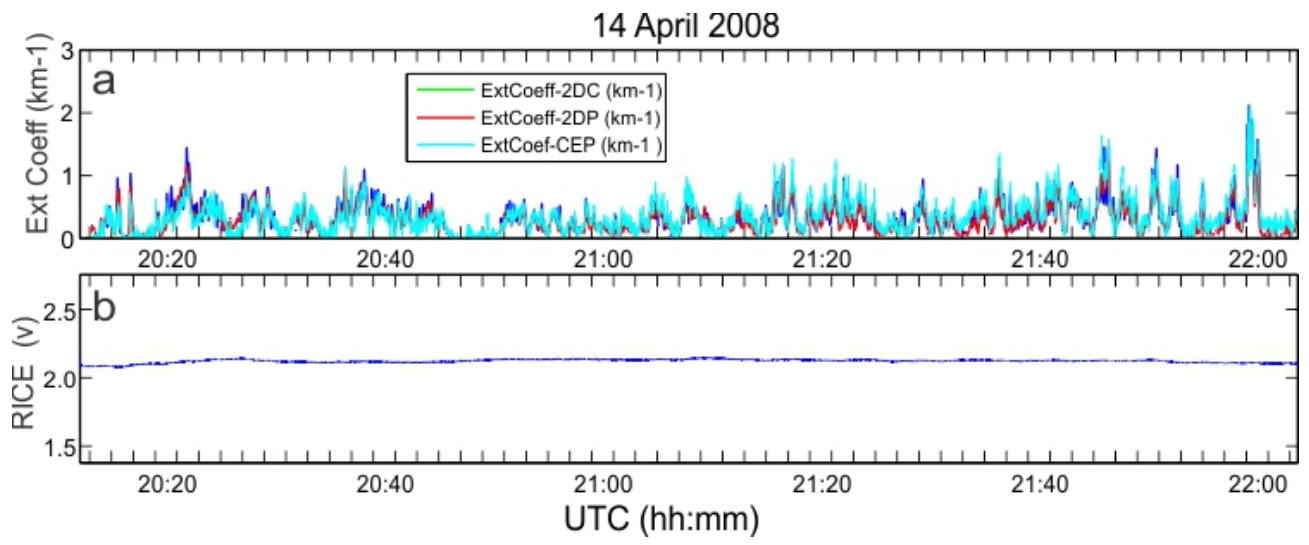

Figure 37. Time series of the measurements of extinction coefficient by the CEP and particle probes OAP-2DC and OAP-2DP (a) and Rosemount Icing Detector signal (b). The measurements were conducted in Cs-As clouds during the ISDAC field campaign in the Cape Barrow region, at $540<\mathrm{P}<680 \mathrm{mb} ;-34<\mathrm{T}<28 \mathrm{C}$, on 14 April 2008.

The scatter-diagrams in Figure 38a show good agreement between extinction coefficient measured by the CEP and that derived from the OAP-2DC. Figure 39a shows the distribution of extinction coefficient versus particle size averaged over the time period in Figure 37. The extinction distribution in Figure 39a was calculated based on the size-to-area conversion (Eq.31) applied to the measured particle size distribution. The distribution in Figure 39a suggests that extinction coefficient is mainly contributed to by 
particles larger than $\sim 300 \mu \mathrm{m}$. Laboratory calibration by opaque dots and glass beads suggest that the instrumental extinction efficiency $Q(D)$ in this size range should be close to 1 as shown in Figure 39b. In this regard, it is anticipated that the extinction coefficient measured by the CEP should be approximately twice lower than that calculated from the imaging probes. However, the comparison between CEP and OAP values in Figures 37a and 38a suggest that the extinction coefficients measured by these two different techniques are in a quite good agreement.
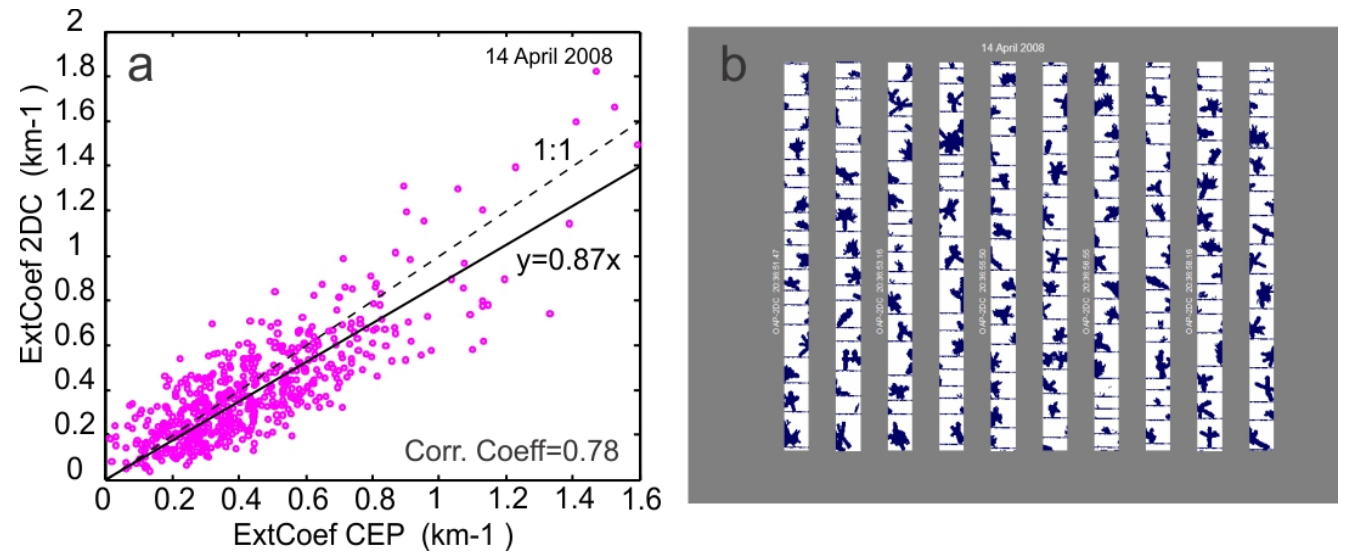

Figure 38. Scatter diagrams of extinction coefficient measured by CEP and OAP-2DC (a) and particle images measured by OAP-2DC (b) for the time interval shown in Figure 37.
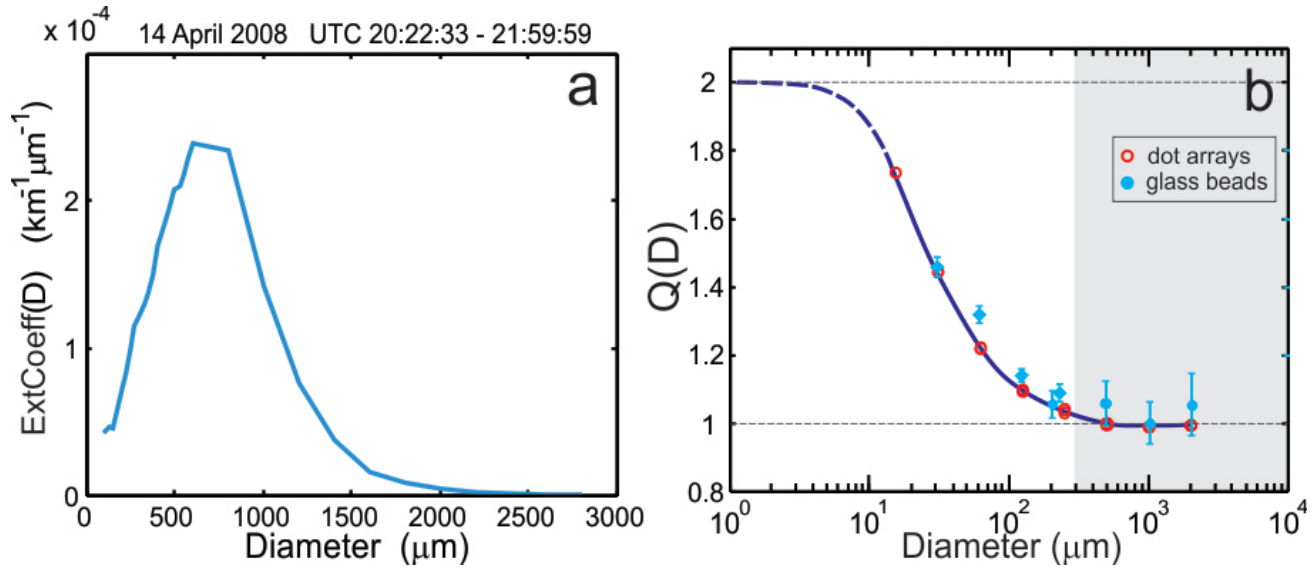

Figure 39. Distribution of extinction coefficient calculated from OAP-2DC and averaged over the time period corresponding to that shown in Figure 37 (a). Gray area on (b) indicates region of the instrumental extinction efficiency corresponding to the size range of ice particle mainly contributing in the extinction coefficient in (a).

Most of the extinction coefficient measurements conducted during ISDAC for ice clouds were collected in optically thin clouds with $\beta<2 \mathrm{~km}^{-1}$. These values of $\beta$ are close to the threshold sensitivity of the CEP $\left(0.2 \mathrm{~km}^{-1}\right)$, and therefore they can be affected by noise. The following case shows measurements for ice clouds obtained during the C3VP project conducted in southern Ontario during the cold season of 20062007 (Barker et al. 2008), with extinction coefficients up to $20 \mathrm{~km}^{-1}$. The diagrams in Figures $40-41$ show good agreement between extinction coefficient measurements by the CEP and imaging probes. Figure 41 shows that ice particles in this cloud were predominantly spatial dendrites. As in the previous case, ice particles with $\mathrm{D}>300 \mu \mathrm{m}$ provide the main contribution to extinction coefficient (Figure $42 \mathrm{a}$ ). 
25 February 2007

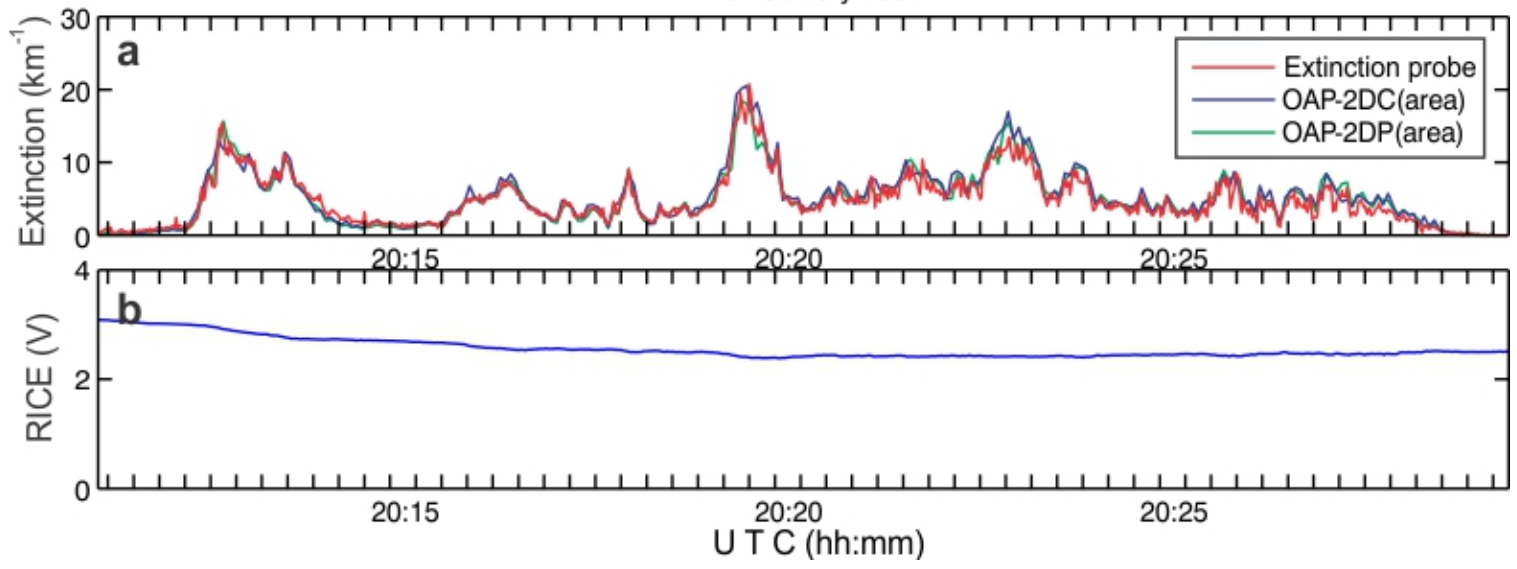

Figure 40. Time series of the measurements of the extinction coefficient by the CEP and particle probes OAP-2DC and OAP-2DP (a) and Rosemount Icing Detector signal (b). The measurements were conducted in Cs-As clouds during the C3VP project in the Ottawa region, at $500<\mathrm{P}<580 \mathrm{mb} ;-24<\mathrm{T}<18 \mathrm{C}$, on 25 February 2007.
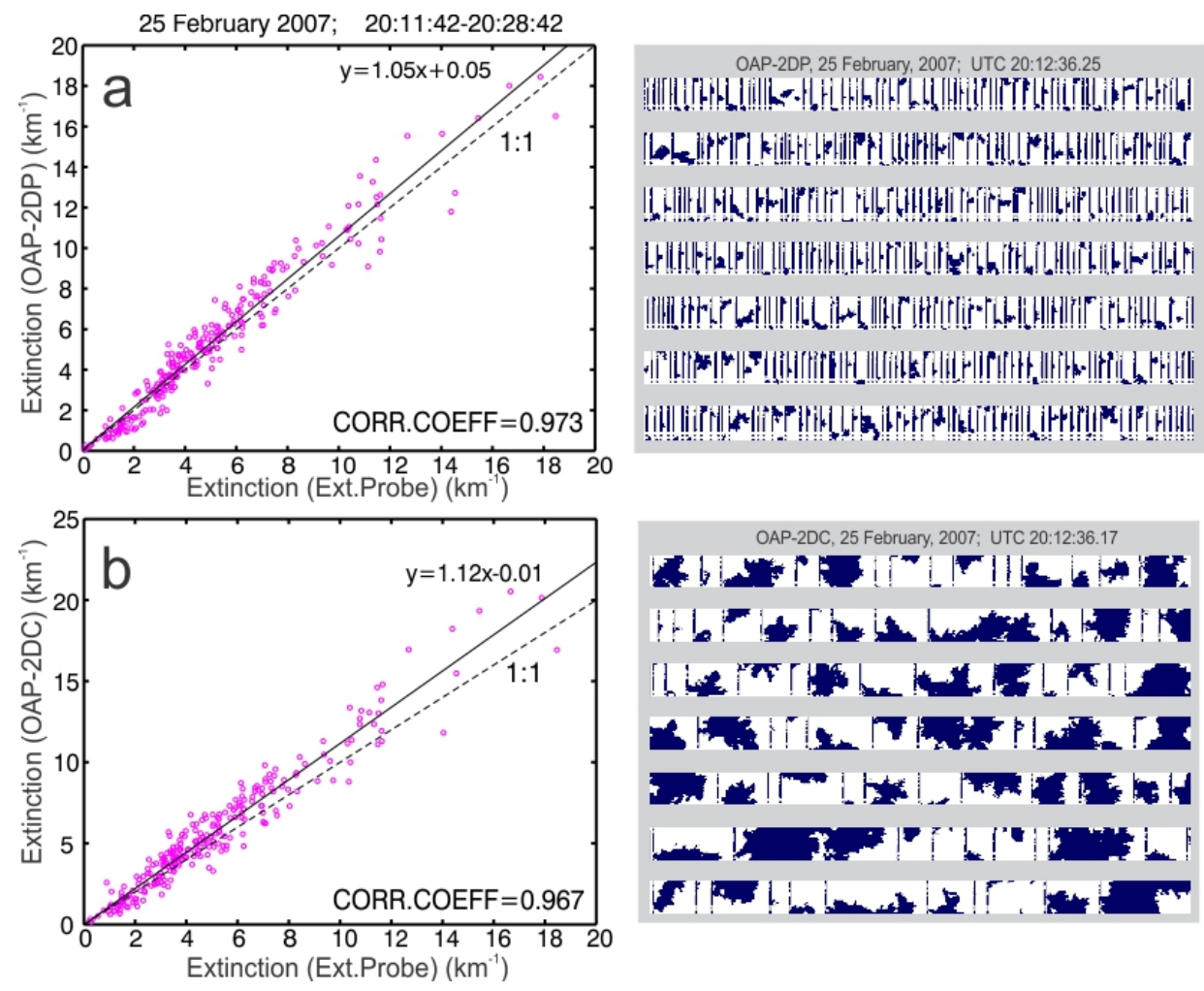

Figure 41. Scatter diagrams of extinction coefficient measured by the CEP and OAP-2DC (a) and OAP2DP (b) during the flight shown in Figure 40. Corresponding 2D images of the particles measured by the OAP-2DC and OAP-2DP are shown on the right. 

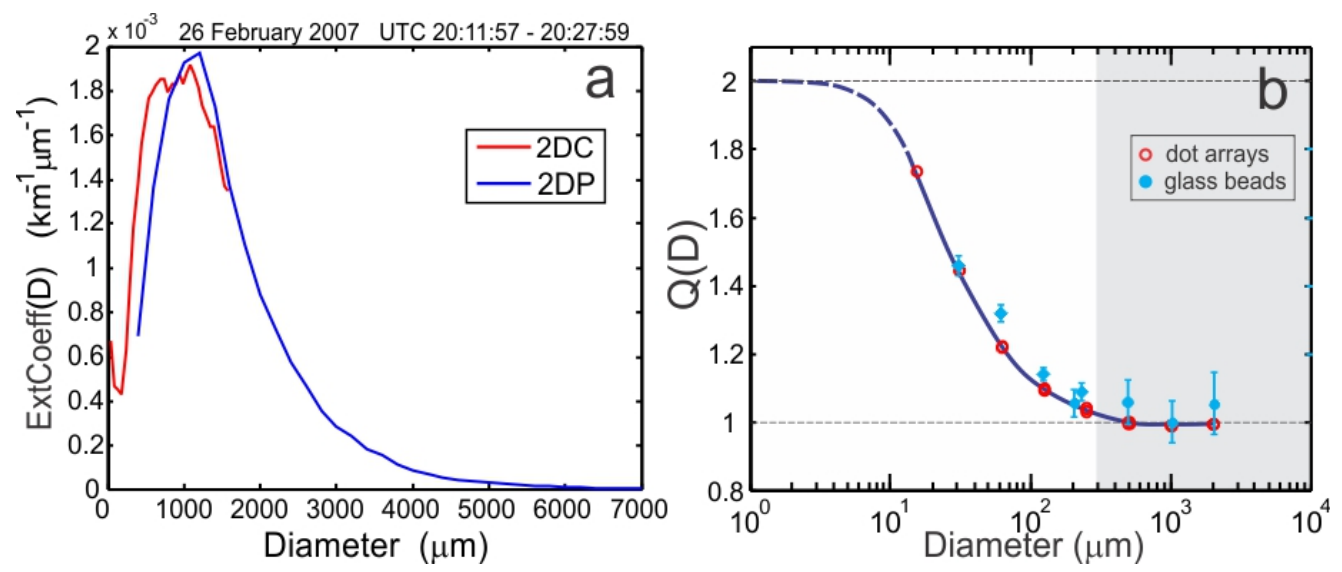

Figure 42. Distribution of extinction coefficient calculated from OAP-2DC/2DP and averaged over the time period corresponding to that shown in Figure 40. Gray area on (b) indicates region of the instrumental extinction efficiency corresponding to the size range of ice particle mainly contributing to extinction coefficient in (a).

\subsection{Feasibility of Corrections}

\subsection{Liquid Clouds}

The laboratory calibrations described in Sections 6.2.4-6.2.5 suggest that CEP instrumental extinction efficiency $Q$ is a unique function of $D$. This enables calculation of $Q(D)$ and the subsequent introduction of corrections to measured extinction coefficient.

Numerous prior measurements have shown that most liquid clouds consist of droplets with $D<10 \mu \mathrm{m}$. The contribution to extinction coefficient by droplets with larger $D$ is expected to be small for the majority of liquid clouds. For droplets $D<10 \mu \mathrm{m}$, extinction efficiency is close to 2, and so CEP inferences of extinction coefficient do not require corrections. However, for cases with larger droplets and precipitation, errors due to forward scattering may reach a factor of two and thus will require correction.

\subsection{Ice Clouds}

It turned out that the agreement between extinction coefficient measured by the CEP and that deduced from the imaging probes is observed for the majority of sampled ice clouds. In all cases, ice particles with $D>300 \mu \mathrm{m}$ are the main contributors to extinction coefficient (see Figure 39a, 42a). In the calculation of extinction coefficient from the imaging probe data in Eq.30 it was assumed that $Q=2$. However, laboratory calibrations by the dot arrays and glass beads suggest that for $D>300 \mu \mathrm{m}$, the CEP instrumental extinction efficiency $Q(D) \sim 1$. Therefore, the CEP in situ measurements of extinction coefficient in ice clouds and its comparisons with the imaging probes appear to contradict the laboratory calibrations. This is because agreement between CEP and imaging probes implies that $Q(D) \sim 2$. The laboratory calibrations of extinction coefficient measured by CEP are expected to be roughly half those calculated from the imaging probes. 


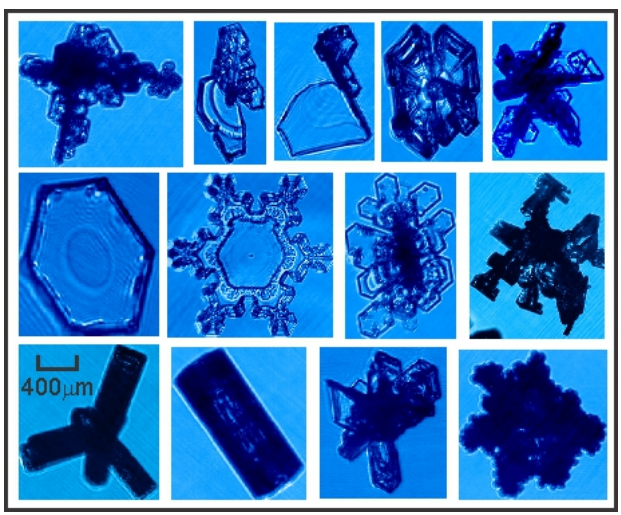

Figure 43. CPI imagery of ice particles.

Potential explanations of the agreement between extinction coefficients measured by the CEP and imaging probes may be related to: (a) underestimation of particle areas by the imaging probes or (b) the fact that ice crystals scatter light in the forward direction in wider angles as compared to that for opaque discs and glass beads with the same linear dimensions.

Korolev et al (1998) showed that imaging probes with coherent illumination (OAP-2DC/2DP/2DG, etc.) are susceptible to overestimation of the measured particle size and area. Therefore, the imaging probe measurements are likely to result in overestimation, rather than underestimation, of extinction coefficient. Thus, explanation (a) does not seem a likely reason for the agreement in extinction coefficient measurements.

Figure 43 shows images of cloud ice particles with different habits. Many of these particles have transparent areas. The images of the particles clearly indicate that the dimensions of the distinct components forming an ice particle (e.g., internal inhomogeneities visible in transmitted light, features forming particle edges, etc.) in some cases are much smaller than the linear dimensions of the entire particle. Therefore, it would be reasonable to hypothesize that the forward scattering by such particles may be significantly affected by these small-scale features, thereby resulting in broadening of the forward-scattering lobe. This effect would result in the increase of instrumental scattering efficiency $Q(D)$ eventually approaching 2 . This consideration is supported by the laboratory measurements of broken glass particles (Section 6.2.7). Large glass particles with transparent parts had $Q(D)>1$ (Figures 23, 24).

This consideration raises a series of important questions. First, do ice particles scatter light like a conglomeration of small particles (Figure 44b) or like an opaque solid (Figure 44c)? Second, what is the effect of the transparent parts of the extinction coefficient? Third, how should the transparent parts be accounted for in extinction coefficient calculations stemming from imaging probe data?
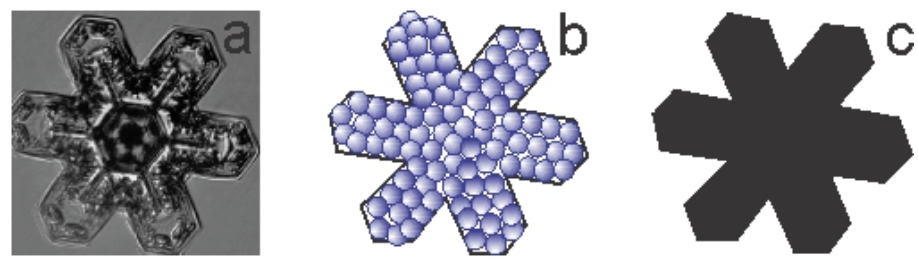

Figure 44. Image of a stellar ice particle showing multiple small-scale features in its internal structure (a). Representation of the ice particle in (a) as a conglomeration of small particles (b) and an opaque solid (c). 
Laboratory experiments described in Section 6.2 along with the frequently observed agreement between extinction coefficient measured by CEP and imaging probes, obtained from in situ measurements, suggest that for ice particles, $Q(D)$ is an ambiguous function of $D$. In other words, information only about linear size $D$ of an ice particle is insufficient to determine instrumental extinction efficiency $Q$. Ideally, a scattering function for ice particles would uniquely address this question. A potentially attractive approach seems to be that ice particle habit along with the particle size may provide a link to the scattering function and $Q$. However, the infinite variety of particle shapes significantly hinders this approach and even makes it impossible if one requires arbitrarily high accuracies.

\subsection{Summary}

The following outcomes have been obtained in the frame of this work:

1. Laboratory calibrations of the CEP by dot arrays and glass beads showed that $Q(D)$ can be considered as a unique function of $D$. This finding confirms that corrections of extinction coefficient measured in liquid clouds are feasible. The corrections of extinction coefficient can be made based on droplet size distributions measured by particle probes (e.g., FSSP, CDP) and the function $Q(D)$ obtained from the laboratory calibrations.

2. For most measurements in liquid clouds during the ISDAC field campaign, the contribution to extinction coefficient by droplets with $D>15 \mu \mathrm{m}$ was relatively small and does not exceed $10-15 \%$. Therefore, most CEP measurements do not require corrections due to forward scattering and thus can be used as reported.

3. It appears that for the case of ice particles, instrumental scattering efficiency $Q$ is not a unique function of particle linear size. At this stage corrections of extinction coefficient measured in ice clouds does not seem feasible. Additional studies are required in this regard.

\subsection{Recommendations for Further Work}

The Cloud Extinction Probe is a new instrument that lacks analogues among existing airborne instruments that attempt to measurement extinction coefficient. The set of experiments described in this work is a first attempt to characterize instrumental scattering efficiency $Q$ from laboratory measurements. The set of tests and calibrations described in this report showed problems relevant to transmissiometeric technique. The results of this work, and the developed methodology, can be used as a starting point for further improvements to existing airborne transmissiometers.

The outcomes of this study bring up a series of important questions, which should be addressed in future studies:

1. Do ice particles scatter light like conglomerations of small particles (Figure 44b) or like an opaque solid (Figure 44c)?

2. What are the effects of the transparent parts of crystals on their extinction coefficient?

3. How should the transparent parts of crystals be treated in extinction coefficient calculations from imaging probe data? 


\subsection{References}

Barker, HW, AV Korolev, DR Hudak, JW Strapp, and KB Strawbridge. 2008. “A comparison between CloudSat and aircraft data for a multilayer, mixed phase cloud system during the Canadian CloudSatCALIPSO Validation Project.” Journal of Geophysical Research 114: D00A16, doi:10.1029/2008JD009971.

Deepak, A, and MA Box. 1978a. "Forward scattering corrections for optical extinction measurements in aerosol media. 1: Monodispersions.” Applied Optics 17: 2900-2908.

Deepak, A, and MA Box. 1978b. "Forward scattering corrections for optical extinction measurements in aerosol media. 1: Polydispersions.” Applied Optics 17: 3169-3176.

Gerber, H, Y Takano, TJ Garrett, and PV Hobbs. 2000. "Nephelometer Measurements of the Asymmetry Parameter, Volume Extinction Coefficient, and Backscatter Ratio in Arctic Clouds.” Journal of the Atmospheric Sciences 57: 3021-3034.

Gumprecht, RO, and CM Sliepevich. 1953. “Scattering light by large spherical particles.” The Journal of Physical Chemistry 57: 90-95.

Kampe, aufm, HJ. 1950. "Visibility and Liquid Water Content in Clouds in the Free Atmosphere.” Journal of the Atmospheric Sciences 7: 54-57.

King, WD, and RJ Handsworth. 1979. “Total droplet concentration and average droplet sizes from simultaneous liquid water content and extinction measurements.” Journal of Applied Meteorology 18: 940-944.

Korolev, AV, JW Strapp, GA Isaac, and AN Nevzorov. 1999. "In situ measurements of effective diameter and effective droplet number concentration.” Journal of Geophysical Research 27: 3993-4003.

Korolev, AV, G Isaac, I Mazin, and H Barker. 2001. "Microphysical properties of continental stratiform clouds.” Quarterly Journal of the Royal Meteorological Society 127: 2117-2151.

Korolev, A. 2008. "New Airborne Extinction Probe.” International Conference on Clouds and Precipitation, 7-11 Aug. 2008, Cancun, Mexico, CD P13.3.

Kosarev, AL, IP Mazin, AN Nevzorov, and VF Shugaev. 1976. “Optical density of clouds.” Cent. Aerol. Obs. (translated) 124: 44-110.

Mazin, IP, AV Korolev, A Heymsfield, GA Isaac, and SG Cober. 2001. "Thermodynamics of Icing Cylinder for Measurements of Liquid Water Content in Supercooled Clouds.” Journal of Atmospheric and Oceanic Technology 18: 543-558.

Nevzorov, AN, and VF Shugaev. 1974. “Aircraft cloud extinction meter.” Cent. Aerol. Obs. (translated) 106: 3-10. 
Nevzorov AN, and VF Shugaev. 1972. "The use of integral parameters for study of cloud microstructure.” Cent. Aerol. Obs. (translated) 101: 32-47.

Ruskin, RR. 1974. "The measurements of cloud elements: the use of transmission and scattering techniques to measure ice and water.” Proceedings of the Symposium on the Measurement of Cloud Elements, W.D. Scott, Ed. NOAA, Tech Memo ERL WMPO-19, 117.

Shifrin, KM. 1988. Physical optics of ocean water. New York: American Institute of Physics.

van de Hulst, HC. 1957. Scattering of light by small particles. New York: Wiley.

Zabrodsky, GM. 1957. "Measurements and some results of study of visibility in clouds.” In Study of Clouds, Precipitation, and Cloud Electrification. Gidrometeizdat, 131-134.

Zmarzly, PM, and RP Lawson. 2000. An Optical Extinctiometer for Cloud Radiation Measurements and Planetary Exploration. Final Report submitted to NASA Goddard Space Flight Center in fulfillment of Contract NAS5-98032, September, 2000, 131 pp. (available from http://www.specinc.com/publications/Extinctiometer Report.pdf).

Weickmann, HK, and HJ aufm Kampe. 1953. "Physical properties of cumulus clouds.” Journal of Atmospheric Sciences 10: 204-211. 


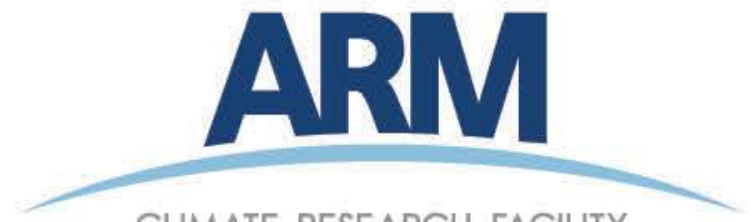

CLIMATE RESEARCH FACILITY

www.arm.gov

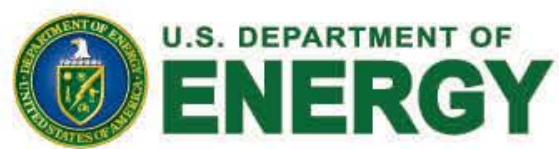

Office of Science 